\title{
Monte Carlo study of a generalized icosahedral model on the simple cubic lattice
}

\author{
Martin Hasenbusch \\ Institut für Theoretische Physik, Universität Heidelberg, \\ Philosophenweg 19, 69120 Heidelberg, Germany
}

(Dated: July 13, 2020)

\begin{abstract}
We study the critical behavior of a generalized icosahedral model on the simple cubic lattice. The field variable of the icosahedral model might take one of twelve vectors of unit length, which are given by the normalized vertices of the icosahedron, as value. Similar to the Blume-Capel model, where in addition to -1 and 1 , as in the Ising model, the spin might take the value 0 , we add in the generalized model $(0,0,0)$ as allowed value. There is a parameter $D$ that controls the density of these voids. For a certain range of $D$, the model undergoes a second-order phase transition. On the critical line, $O(3)$ symmetry emerges. Furthermore, we demonstrate that within this range, similar to the Blume-Capel model on the simple cubic lattice, there is a value of $D$, where leading corrections to scaling vanish. We perform Monte Carlo simulations for lattices of a linear size up to $L=400$ by using a hybrid of local Metropolis and cluster updates. The motivation to study this particular model is mainly of technical nature. Less memory and CPU time are needed than for a model with $O(3)$ symmetry at the microscopic level. As the result of a finite-size scaling analysis we obtain $\nu=0.71164(10), \eta=0.03784(5)$, and $\omega=0.759(2)$ for the critical exponents of the three-dimensional Heisenberg universality class. The estimate of the irrelevant renormalization group eigenvalue that is related with the breaking the $O(3)$ symmetry is $y_{\text {ico }}=-2.19(2)$.
\end{abstract}




\section{INTRODUCTION}

In the neighborhood of a second-order phase transition, thermodynamic quantities diverge, following power laws. For example, the correlation length behaves as

$$
\xi=a_{ \pm}|t|^{-\nu}\left(1+b_{ \pm}|t|^{\theta}+c t+\ldots\right)
$$

where $t=\left(T-T_{c}\right) / T_{c}$ is the reduced temperature. The subscript \pm of the amplitudes $a_{ \pm}$ and $b_{ \pm}$indicates the high $(+)$and the low $(-)$temperature phase, respectively. There are non-analytic or confluent and analytic corrections. The leading ones are explicitly given in eq. (1). In the literature, the exponents associated with the specific heat, the magnetization, and the magnetic susceptibility are denoted by $\alpha, \beta$, and $\gamma$, respectively. The exponent of the magnetization at the critical temperature for a non-vanishing external field is denoted by $\delta$. The exponent $\eta$ governs the behavior of the two-point function at the critical point. For the precise definition of these exponents and relations between them see for example section 1.3 of the review [1]. Second-order phase transitions are grouped into universality classes. For all transitions within such a class, critical exponents assume identical values. Also correction exponents such as $\theta=\omega \nu$ are universal. Universality classes are characterized by the symmetry properties of the order parameter at criticality, the range of the interaction and the spatial dimension of the system. For reviews on critical phenomena see, for example, $[1] 4]$.

Note that in general the symmetry properties of the order parameter can not be naively inferred from the microscopic properties of the system. In particular, a symmetry might emerge that is not present in the classical Hamiltonian. For example, in a binary mixture, the two components are not related by a $\mathbb{Z}_{2}$ symmetry. However, in case the mixing-demixing transition is of second order, it belongs to the Ising universality class, which is characterized by a $\mathbb{Z}_{2}$ symmetry of the order parameter.

In the present work we are aiming at a precise determination of the critical exponents of the three-dimensional Heisenberg universality class. To this end, we study a generalized icosahedral model on the simple cubic lattice. The field variable takes the normalized vertices

of the icosahedron as values. In addition $(0,0,0)$ might be assumed. In the following we refer to field variables assuming this value as voids. The idea to use a discrete subset of the sphere as values of the field variable is rather old [5, 6], however received little attention. Note that the field variable is also referred to as spin. The model has a parameter $D$ that 
controls the density of voids. For a certain range of this parameter, the model undergoes a second-order phase transition. Our numerical data show that at the phase transition, the model is in the domain of attraction of the $O(3)$-invariant fixed point. Hence, the model shares the universality class of the three-dimensional $O(3)$-invariant Heisenberg model. On the critical line, similar to the Blume-Capel model, the amplitude of the leading correction depends on $D$. We demonstrate that there is one value $D^{*}$ of $D$, where leading corrections to scaling vanish. We refer to the model at $D \approx D^{*}$ as improved model. Simulating an improved model allows us to get more accurate estimates of universal quantities at a given budget of CPU time. For a brief discussion and references on improved models in general see [7].

As discussed below in more detail, a perturbation with the symmetry properties of the icosahedron is irrelevant at the $O(3)$-invariant fixed point. In the Appendix $\mathrm{B}$ we determine the corresponding renormalization group $(\mathrm{RG})$ eigenvalue $y_{i c o}=-2.19(2)$. Likely, analogous to the case of the clock model discussed in ref. [7], the perturbation is dangerously irrelevant. Meaning that in the low-temperature phase, in the thermodynamic limit, the spontaneous magnetization might only assume one of the 12 directions that are preferred by the Hamiltonian. Note, however, that this does not affect the finite size scaling study at the critical point that we perform here.

Our motivation to study this model is that simulations take less CPU time than for an $O(3)$-invariant model as, for example, the $\phi^{4}$ model simulated in refs. [8 10] and less memory is needed to store the field variables. The idea of the present work is similar to that of ref. [7], where we studied the $(q+1)$-state clock model. In addition to the $q$ values with unit length, the value $(0,0)$ might be assumed by the field variable. In the case of the $(q+1)$-state clock model, an $O(2)$-invariant model can be approached by taking the limit $q \rightarrow \infty$. In contrast, here we are restricted to the Platonic solids.

The Heisenberg universality class describes the critical behavior of isotropic magnets, for instance the Curie transition in isotropic ferromagnets such as $\mathrm{Ni}$ and $\mathrm{EuO}$, and of antiferromagnets such as $\mathrm{RbMnF}_{3}$ at the Néel transition point. A summary of experimental results for critical exponents is given in the tables 24 and 25 of the review [1]. An example for a more recent experimental study is ref. [11]. In table 2 of [11] estimates for the critical exponents $\beta, \gamma, \delta$ and $\alpha$ are presented for four different materials. To get an idea of the accuracy that is achieved let us pick out two results for GdScGe: $\alpha=-0.134 \pm 0.005$ 
for the exponent of the specific heat and $\delta=4.799 \pm 0.006$ for the critical exponent of the magnetization on the critical isotherm. Using scaling relations these exponents can be converted to $\nu=0.7113(17)$ and $\eta=0.0347(11)$, which are the exponents given in table I] below.

The three-dimensional Heisenberg universality class has been studied by using various theoretical approaches. Well established field theoretic methods are the $\epsilon$-expansion and the perturbation theory in three dimensions fixed. In order to extract numerical estimates for critical exponents, various resummation schemes are discussed in the literature. As examples we give in table \ the estimates obtained in ref. [12]. Recently there has been progress in the $\epsilon$-expansion and the six-loop coefficient has been computed for the $O(N)$-invariant $\phi^{4}$ theory [13]. In table [, we give the results of the resummation used in ref. [13] based on the five- and six-loop $\epsilon$-expansion. The five- and six-loop estimates are consistent. Note however that for the five-loop resummation, the estimate of the error differs at lot between ref. [12] and ref. [13]. For a discussion of the resummation schemes used, we refer the reader to refs. [12, 13]. The $\epsilon$-expansion has been extended to seven-loop [14]. However no numerical estimates for critical exponents have been computed so far.

Great progress has been achieved recently by using the so called conformal bootstrap (CB) method. In particular in the case of the three-dimensional Ising universality class, the accuracy that has been reached for critical exponents clearly surpasses that of other theoretical methods. See ref. [15] and references therein. Very recently also highly accurate estimates were obtained for the XY universality class [16], surpassing the accuracy of results obtained by lattice methods. Still for the Heisenberg universality class [17], the estimates are less precise than those obtained by other methods.

Considerable progress has also been achieved by using the functional renormalization group method. In ref. [18] the authors have computed the critical exponents $\nu, \eta$ and the correction exponent $\omega$ for various values of $N$. In the tables IV, V, VI and VII of [18] the authors summarize their results and compare them with estimates obtained by other methods for $N=1,2,3$, and 4, respectively. A good agreement with the results of the conformal bootstrap is found. The same holds for the comparison with estimates obtained by studying lattice models. In table 1 we report the estimates obtained for $N=3$.

Finally we report results obtained for the $O(3)$-invariant $\phi^{4}$ model on the simple cubic lattice. Note that there exists a value $\lambda^{*}$ of the coupling constant $\lambda$ of this model such 
TABLE I. We give a selection of theoretical results for the critical exponents $\nu$ and $\eta$ and the exponent $\omega$ of the leading correction to scaling for the three-dimensional Heisenberg universality class obtained by various methods. For a more comprehensive summary see for example table 23 of ref. [1]. For the definition of the acronyms and a discussion see the text.

\begin{tabular}{cccccc}
\hline Ref. & method & year & $\nu$ & $\eta$ & $\omega$ \\
\hline$[12]$ & 3D-exp. & $19980.7073(35)$ & $0.0355(25)$ & $0.782(13)$ \\
{$[12]$} & $\epsilon$-exp. 5l & $19980.7045(55)$ & $0.0375(45)$ & $0.794(18)$ \\
{$[13]$} & $\epsilon$-exp. 5l & $20170.7056(16)$ & $0.0382(10)$ & $0.797(7)$ \\
{$[13]$} & $\epsilon$-exp. 61 & $20170.7059(20)$ & $0.0378(5)$ & $0.795(7)$ \\
{$[17]$} & CB & $20160.7121(28)$ & $0.03856(124)$ & - \\
{$[18]$} & NRG & $20200.7114(9)$ & $0.0376(13)$ & $0.769(11)$ \\
{$[8]$} & MC & $20010.710(2)$ & $0.0380(10)$ & - \\
{$[9]$} & MC+HT & $20020.7112(5)$ & $0.0375(5)$ & - \\
{$[9,10]$} & MC+HT & $20020.7117(5)$ & $0.0378(5)$ & - \\
{$[10]$} & MC & $20110.7116(10)$ & $0.0378(3)$ & - \\
[9], present work & MC+HT, $\phi^{4}$ & 2020 & $0.7116(2)$ & $0.0378(3)$ & - \\
present work & MC, $\phi^{4}$ & 2020 & $0.71164(25)$ & $0.03782(10)$ & - \\
present work & MC, icosahedral $20200.71164(10)$ & $0.03784(5)$ & $0.759(2)$ \\
\hline
\end{tabular}

that the leading correction to scaling vanishes. In ref. [8] a finite size scaling analysis of Monte Carlo (MC) data was performed. In ref. [9] both Monte Carlo simulations and the high temperature (HT) series expansion were used. In particular, the analysis of the HT series by using integral approximants [19] is biased by using the estimates of the inverse critical temperature and $\lambda^{*}=4.6(4)$ obtained by Monte Carlo simulations. In ref. [10] we mainly focused on the RG-eigenvalues of anisotropic perturbations at the $O(N)$-invariant fixed point. As a byproduct, we get the revised estimate $\lambda^{*}=5.2(4)$. The values quoted for refs. [9, 10] are obtained by inserting this value into eqs. $(13,14,19)$ of ref. [9]. Next we report the results of Monte Carlo simulations that we discuss in appendix $\mathrm{A}$. The estimate of the inverse critical temperature and $\lambda^{*}$ are used to bias the HT analysis of ref. [9]. Finally we report the results obtained from the finite size scaling study of the generalized icosahedral 
model. By using a hybrid of local and cluster algorithms we simulated lattices of a linear size up to $L=400$. It is virtually impossible to give a comprehensive summary of the vast literature on the subject. For a more extensive summary see for example table 23 of ref. [1].

We notice that our results for $\nu$ and $\eta$ obtained for the generalized icosahedral model are fully consistent with those that were obtained for the $\phi^{4}$ model on the simple cubic lattice. Our results are also consistent with but more precise than those of refs. [17, 18] obtained by using the conformal bootstrap method and the functional renormalization group method, respectively.

Comparing with the results obtained from the resummation of the $\epsilon$-expansion we see clear differences. Our result for $\nu$ is larger than that obtained in ref. [13] by about three times the error that is quoted. The result for the correction exponent $\omega$ obtained in ref. [13] is roughly by five times the error that is quoted larger than ours.

The outline of the manuscript is the following: In section \we define the model and the observables that we measured. Furthermore, we summarize theoretical results on subleading corrections to scaling. In section [II we discuss the Monte Carlo algorithm used in the simulations and outline our approach to the analysis of the data. In section IV we analyze the data and present the results for the fixed point values of phenomenological couplings, inverse critical temperatures, the correction exponent $\omega$, and the critical exponents $\nu$ and $\eta$. In section $\nabla$ we conclude and give an outlook. In Appendix $₫$ we discuss our results for the three-component $\phi^{4}$ model on the simple cubic lattice. Finally, in Appendix B we determine the RG-exponent $y_{i c o}$ related with the breaking of the $O(3)$ symmetry.

\section{THE MODEL}

We consider a simple cubic lattice. A site is given by $x=\left(x_{0}, x_{1}, x_{2}\right)$, where $x_{i} \in$ $0,1,2, \ldots, L_{i}-1$. In our simulations $L_{0}=L_{1}=L_{2}=L$ throughout and periodic boundary conditions are imposed. The model is analogous to the $(q+1)$-state clock model discussed in ref. [7]. In the case of the $(q+1)$-state clock model the spins $\vec{s}_{x}$ take either values on the unit circle or assume the value $(0,0)$. Here the circle is replaced by the two-sphere. In particular, the spin $\vec{s}_{x}$ might take one of the thirteen values $\vec{v}_{m}$ tabulated below:

$$
(0,0,0), z(0, \pm 1, \pm \phi), \quad z( \pm 1, \pm \phi, 0), \quad z( \pm \phi, 0, \pm 1)
$$


where $\phi=\frac{1}{2}(1+\sqrt{5})$ is the golden ratio and $z=1 / \sqrt{1+\phi^{2}}=1 / \sqrt{2+\phi}$. The twelve vectors with unit length are the normalized vertices of the icosahedron. See for example eq. (A.20) of ref. [20], which is eq. (40) of the preprint version. An alternative choice is given in eq. (A.9), corresponding to eq. (29) of the preprint version. In our simulation program the field variables are stored by using the label $m \in\{0,1,2, \ldots, 12\}$, where $\vec{v}_{0}=(0,0,0)$ and $m \in\{1,2, \ldots, 12\}$ are assigned to the vectors of unit length.

In the following we shall refer to the model as generalized icosahedral model. The reduced Hamiltonian is given by

$$
\mathcal{H}=-\beta \sum_{\langle x y\rangle} \vec{s}_{x} \cdot \vec{s}_{y}-D \sum_{x} \vec{s}_{x}^{2}-\vec{H} \sum_{x} \vec{s}_{x}
$$

where $\langle x y\rangle$ denotes a pair of nearest neighbor sites on the simple cubic lattice. We introduce the weight factor

$$
w\left(\vec{s}_{x}\right)=\delta_{0, \vec{s}_{x}^{2}}+\frac{1}{12} \delta_{1, \vec{s}_{x}^{2}}
$$

that gives equal weight to $(0,0,0)$ and the collection of the 12 values with $\left|\vec{s}_{x}\right|=1$. Now the partition function can be written as

$$
Z=\sum_{\{\vec{s}\}} \prod_{x} w\left(\vec{s}_{x}\right) \exp (-\mathcal{H})
$$

where $\{\vec{s}\}$ denotes a configuration of the field.

The reduced Hamiltonian (3) and the weight (44) are the same as for the $(q+1)$-clock model defined in section II of ref. [7]. The two models only differ in the set of allowed values of the field variables. Note that in the limit $D \rightarrow \infty$ the value $(0,0,0)$ is completely suppressed. In the following we consider a vanishing external field $\vec{H}=(0,0,0)$ throughout.

\section{A. The quantities studied}

The most important quantities are dimensionless quantities $R_{i}$ that are also called phenomenological couplings. In particular we study the ratio of partition functions $Z_{a} / Z_{p}$, where $a$ denotes a system with anti-periodic boundary conditions in one of the directions and periodic ones in the remaining two directions, while $p$ denotes a system with periodic boundary conditions in all directions. Furthermore, we study the second moment correlation length over the linear lattice size $\xi_{2 n d} / L$, the Binder cumulant $U_{4}$ and its generalization 
$U_{6}$. The exponent of the correlation length is determined by studying the finite size scaling behavior of the slopes of dimensionless quantities. The critical exponent $\eta$ is obtained from the finite size scaling behavior of the magnetic susceptibility $\chi$. These quantities are defined for example in section II B of ref. [7]. In our analysis, the observables are needed as a function of the inverse temperature $\beta$ for a neighborhood of the inverse critical temperature $\beta_{c}$. To this end, we simulate at $\beta_{s}$, which is a preliminary estimate of $\beta_{c}$ and compute the coefficients of the Taylor expansion in $\left(\beta-\beta_{s}\right)$ up to third order.

\section{B. Subleading corrections to scaling}

Analyzing our data, we use prior information on subleading corrections to scaling. These corrections are due to $O(N)$-invariant perturbations of the fixed point and perturbations that break the $O(N)$-invariance. Let us first discuss the former. In section III A of ref. [7] we conclude, based on the literature, that there should be only a small dependence of the irrelevant RG-eigenvalues on $N$. Therefore the discussion of section III A of ref. [7] should apply to the present case $N=3$ at least on a qualitative level. In particular, we regard the subleading correction exponent $\omega_{2}=1.78(11)$ that we assumed in refs. [9, 10] as an artifact of the scaling field method [21]. Instead, the most important subleading correction should be due to the breaking of the rotational symmetry by the simple cubic lattice. Following ref. [22], the associated correction exponent is $\omega_{N R} \approx 2.02$.

Now let us turn to the corrections caused by the breaking of the $O(3)$-invariance. A good starting point of the discussion is provided by ref. [20]. In section 2, polynomials are constructed that are invariant under the action of the discrete symmetry groups related with the Platonic solids and belong to an irreducible representation of the $O(3)$ group. Hence, they have a well defined $O(3)$ spin $n$. In eq. (3) of ref. [20], polynomials associated with the tetrahedron, the cube, and the icosahedron are given. These are associated with the spin $n=3,4$, and 6 . Note that the tetrahedron is self-dual, the octahedron is dual to the cube and the dodecahedron is dual to the icosahedron. There are no further Platonic solids in three dimensions. Note that dual Platonic solids share the symmetry properties. Hence, using the dodecahedron instead of the icosahedron as approximation of the sphere should result in the same irrelevant RG-exponent.

In the case of a two-dimensional system, as discussed in ref. [20], these perturbations 
of the $O(3)$-symmetry are relevant. In particular, the icosahedral model undergoes a phase transition at a finite temperature, while the $O(3)$-symmetric model is asymptotically free and hence no phase transition occurs at a finite temperature.

In ref. [10] we determined the RG-exponents $y_{n}=1.7906(3), 0.9616(10)$, and 0.013(4) for $N=3$ and three spatial dimensions for spin $n=2,3$, and 4, respectively. Hence, for example a cubical model could not be used to study the properties of the $O(3)$ invariant fixed point, since the perturbation is relevant. We could not find a result for $N=3$ and $n=6$ in the literature. However it is interesting to note that the estimates of $y_{n}$ for $n=2$, 3 , and 4 for $N=3$ are well approximated by the average of the corresponding values for $N=2$ and 4. In refs. [23, 24] the estimates $y_{6}=-2.509(7)$ and $-2.069(7)$ are given for $N=2$ and 4 , respectively. Therefore we would expect $y_{6} \approx-2.29$ for $N=3$. In appendix B we find $y_{6}=-\omega_{i c o}=-2.19(2)$. For a discussion of Platonic solids related with stable fixed points in three dimensions, see ref. [25].

There are also corrections that are not related to irrelevant scaling field, such as the analytic background of the magnetic susceptibility. Effectively, it behaves as a correction with the exponent $2-\eta$. For a more comprehensive discussion of subleading corrections see section III of [7].

\section{SIMULATION ALGORITHM}

The algorithm used is very similar to the one discussed in section IV of ref. [7]. We simulated the model by using a hybrid of local updates and cluster updates [26]. In the case of the cluster algorithm, we have implemented the single cluster algorithm [27] and the wall cluster algorithm [28].

\section{A. Local Metropolis updates}

In order to speed up the local updates, in ref. [7] we tabulate the contribution to the Boltzmann factor by pairs

$$
B(m, n)=\exp (\beta \vec{s}(m) \cdot \vec{s}(n))
$$

and its inverse $B^{-1}(m, n)$, where $m$ and $n$ are the labels of the values of the spins. In order to adapt the implementation of the local updates of ref. [7] to the present case, we just had 
to plug in the scalar products $\vec{s}(m) \cdot \vec{s}(n)$ for the vectors given in eq. (2).

Similar to ref. [7] we have used two versions of the Metropolis update that differ in the choice of the proposal. In the first version we always propose $\vec{s}_{x}{ }^{\prime}=(0,0,0)$ if $\left|\vec{s}_{x}\right|=1$ and, with equal probability, one of the 12 values with unit length if $\vec{s}_{x}=(0,0,0)$.

In the second version, the proposal does not depend on $\vec{s}_{x}$. With probability $1 / 2$ we propose $\vec{s}_{x}{ }^{\prime}=(0,0,0)$ and with probability $1 / 24$ one of the 12 values with unit length. The second choice is used in addition to the first one, since we were not able to prove ergodicity for the first one.

\section{B. The cluster algorithms}

Using the cluster algorithm, a spin is potentially changed by a reflection at one of the 15 symmetry planes of the icosahedron. The reflection can be written as

$$
\vec{s}^{\prime}=\vec{s}-2(\vec{r} \cdot \vec{s}) \vec{r}
$$

where $\vec{r}$ is a unit vector perpendicular to the symmetry plane. Being too lazy to search the literature, we computed the possible values of $\vec{r}$ by using a simple Python program. First we define for all pairs of vertices $\vec{v}_{i}$ of the icosahedron a candidate

$$
\vec{c}_{i j}=\frac{\vec{v}_{i}+\vec{v}_{j}}{\left|\vec{v}_{i}+\vec{v}_{j}\right|} .
$$

Then we checked that the candidate is indeed a reflection. Finally we search for multiple identifications of the same reflection. The remaining results for $\vec{r}$ can be grouped in 5 triples of vectors that are mutually orthogonal:

$$
\begin{array}{ccc}
(1,0,0) & (0,1,0) & (0,0,1) \\
(-a, 1 / 2, b) & (1 / 2, b, a) & (b, a,-1 / 2) \\
(a, 1 / 2, b) & (-1 / 2, b, a) & (b,-a, 1 / 2) \\
(1 / 2, b,-a) & (b, a, 1 / 2) & (a,-1 / 2, b) \\
(-b, a, 1 / 2) & (1 / 2,-b, a) & (a, 1 / 2,-b),
\end{array}
$$

where $a=\phi / 2, b=\phi / 2-1 / 2$ and $\phi=\frac{1}{2}(1+\sqrt{5})$ is the golden ratio.

As usual, the cluster algorithm is characterized by the delete probability of a pair of nearest neighbor sites [27]

$$
p_{d}(x, y)=\min \left[1, \exp \left(-2 \beta\left[\vec{r} \cdot \vec{s}_{x}\right]\left[\vec{r} \cdot \vec{s}_{y}\right]\right)\right]
$$


Below we shall refer to a pair of nearest neighbor sites as link. A link $\langle x y\rangle$ is deleted with probability $p_{d}(x, y)$. Otherwise it is frozen.

In the program, we computed all possible values of $p_{d}(x, y)$ before the simulation is started, and store the results in a $15 \times 13 \times 13$ array of double precision floating point values.

Different cluster algorithms are characterized by the way clusters are selected. In the Swendsen-Wang algorithm [26], the whole lattice is decomposed into clusters of sites that are connected by frozen links. In the Swendsen-Wang algorithm, a cluster is flipped with probability 1/2. Flipping means that for all sites within a cluster, the reflection, eq. (77), is performed. In the case of the single cluster algorithm [27], one site of the lattice is randomly selected. Then only the cluster that contains this site is constructed. This cluster is flipped with probability 1. In the wall cluster algorithm [28], instead of a single site a plane perpendicular to one of the lattice axis is chosen. The position on this axis is randomly chosen. Then all clusters that contain sites within this plane are constructed and flipped with probability one. The measurement of $Z_{a} / Z_{p}$ is discussed in the Appendix A 2 of ref. [29].

\section{The update cycle}

The update steps discussed above are compounded into a complete update cycle. Below we give a piece of pseudo $\mathrm{C}$-code that represents the cycle that is used in our simulations:

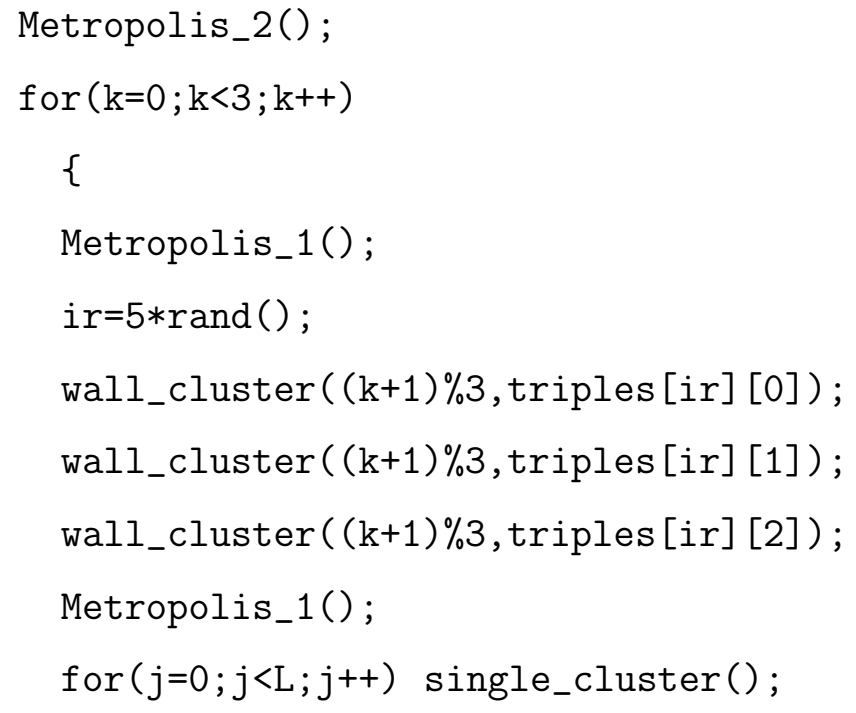




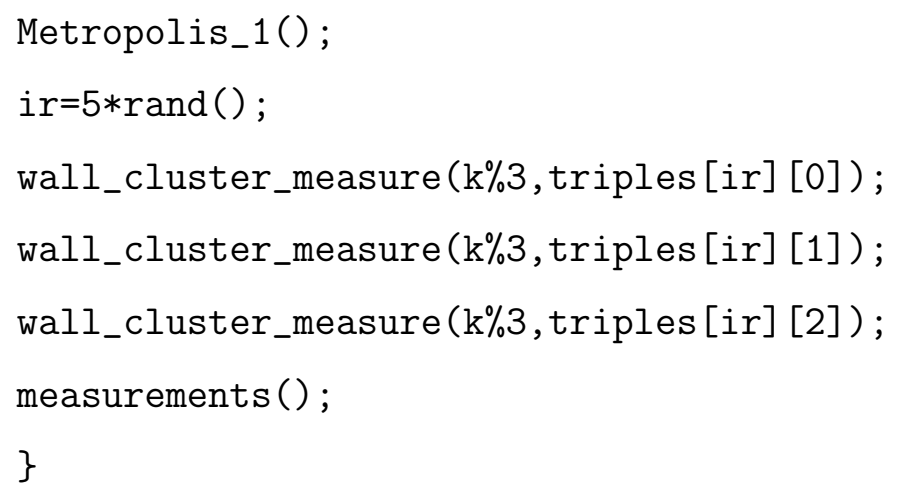

Here Metropolis_1() and Metropolis_2() are sweeps, using the first and second type of the Metropolis update discussed in section IIIA. The single cluster update is given by single_cluster(). For each call, the reflection $\vec{r}$ and the site, where the cluster is started are randomly selected with a uniform distribution. wall_cluster(k\%3,triples [ir] [i]) is a wall cluster update. The first argument selects the spatial direction. The array triples [ir] [i] determines which reflection $\vec{r}$ is chosen for the cluster update. The first index ir selects the set of mutually orthogonal $\vec{r}$ that is taken. Then within such a set we run through all three $\vec{r}$. The wall cluster update is either called just for updating the configuration or, in the case of wall_cluster_measure to perform a measurement of the ratio of partition functions $Z_{a} / Z_{p}$ in addition.

Most of the simulations were performed by using the update cycle discussed above. Below we shall refer to this cycle as cycle A. At a certain stage of the simulation, we realized that for some of the quantities it is more efficient to measure more frequently. Therefore we skipped the wall cluster updates without measurement and reduced the number of single cluster updates from $L$ to $L / 2$. Furthermore one of the Metropolis_1() sweeps is skipped. Below we shall refer to this cycle as cycle B.

We implemented the code in standard $\mathrm{C}$ and used the SIMD-oriented Fast Mersenne Twister algorithm [30] as random number generator.

Since the program is essentially the same as the one used to simulate the $(q+1)$-state clock model, the CPU-times needed for the update of a single site, are identical to those quoted in section IV $\mathrm{C}$ of ref. [7]: Our Metropolis update type one requires $1.2 \times 10^{-8} \mathrm{~s}$ per site. In the case of the single cluster update about $3.8 \times 10^{-8}$ s per site are needed. These timings refer to running the program on a single core of an $\operatorname{Intel}(\mathrm{R}) \mathrm{Xeon}(\mathrm{R}) \mathrm{CPU}$ E3-1225 v3. Compared with the simulation of the $O(3)$-symmetric $\phi^{4}$ model on the simple 
cubic lattice discussed below in appendix $\underline{\mathrm{A}}$, we roughly gain a factor of three.

\section{General remarks on the analysis of the data}

The quantities that we study follow a power law that is subject to corrections

$$
A(L)=a L^{u}\left(1+\sum_{i} c_{i} L^{-\epsilon_{i}}\right)
$$

where $L$ is the linear size of the lattice. By using Monte Carlo simulations, we obtain estimates of $A(L)$ that have statistical errors. Mostly we intend to determine the exponent $u$, which is either the RG-exponent of the thermal scaling field $y_{t}=1 / \nu$ or $2-\eta$ here. The amplitudes $a$ and $c_{i}$ are in general unknown. In the case of the correction exponents we have some prior knowledge. This is gained by theoretical considerations or the analysis of other numerical data, as discussed in section $\llbracket \mathrm{B}$ above. We denote the correction exponents in eq. (10) by $\epsilon_{i}$, since not all are related to a single irrelevant scaling field. The correction exponents of irrelevant scaling fields are given by irrelevant RG-exponents $\omega_{i}=-y_{i}$. Performing least-square fits, we need ansätze that contain only a few free parameters. Hence, the series of corrections in eq. (10) has to be truncated. In our case there is the leading correction with the exponent $\omega=0.759(2)$, see eq. (21) below. Extracting the critical exponents $\nu$ and $\eta$, we consider $D \approx D^{*}$ and on top of that improved observables that are constructed such that the leading correction is suppressed. Therefore it is safe to ignore the leading correction. As discussed in section IIB there are a number of different corrections with $\epsilon_{i} \approx 2$. These are the analytic background of the magnetic susceptibility that effectively corresponds to $\epsilon_{1}=2-\eta$, the violation of the rotational symmetry by the simple cubic lattice $\omega_{N R} \approx 2.02$, and $\omega_{i c o}=2.19(2)$ related to the breaking of the $O(3)$ symmetry. In the case of the slopes that are used to determine $\nu$ there is also $\omega+1 / \nu \approx 2.164$. In principle there is an infinite series of corrections with increasing correction exponents. Since we can deal only with a few free parameters in fits, the sequence has to be truncated at some stage. Even the different corrections with an exponent $\epsilon_{i} \approx 2$ have to be represented by a single or by two effective correction terms. Hence in general the ansatz will never perfectly represent the data. Therefore in addition to the statistical error there is a systematic one that is caused by this imperfection. With increasing linear lattice size $L$, the magnitude

of corrections decreases. If one would consider the linear lattice sizes $L_{\min } \leq L \leq c L_{\min }$, 
where $c>1$, then the estimate of the exponent $u$ would converge with increasing $L_{\min }$, up to the statistical error, to the true answer. Of course, the CPU time that is available sets an upper limit to $c L_{\text {min }}$. Since we would like to squeeze out most from the data we proceed in a different way, similar to most analyses in the literature, all data with $L \geq L_{\min }$ are taken into account. The quality of the fit is measured as usual by

$$
\chi^{2}=\sum_{j}\left[\left(f\left(x_{j},\{p\}\right)-y_{j}\right) / \sigma_{j}\right]^{2},
$$

where $f$ is the ansatz and $\{p\}$ the parameters of the ansatz. In our case, $x_{j}$ are the linear lattice sizes, $y_{j}$ the numerical estimates of the observable and $\sigma_{j}$ its statistical error. In some of the fits below we consider several observables jointly. In this case

$$
\chi^{2}=r C^{-1} r^{T},
$$

where $C$ is the covariance matrix and $r_{j}=y_{j}-f\left(x_{j},\{p\}\right)$. Note that now $x_{j}$ refers to the linear lattice size and the type of the observable. We also perform joint fits for several values of $D$. Then $x_{j}$ also refers to $D$. A fit usually is regarded as acceptable if $\chi^{2} /$ d.o.f. $\approx 1$, where d.o.f. is the number of degrees of freedom. Furthermore we consider the goodness-of-fit $Q=\Gamma_{i n c}^{u p}\left(\right.$ d.o.f. $\left./ 2, \chi^{2} / 2\right)$, where $\Gamma_{i n c}^{u p}$ is the regularized upper incomplete gamma-function. For a Gaussian distribution of the numerical estimates $y_{j}, Q$ gives the probability that, assuming that the ansatz is correct, $\chi^{2}$ is equal to or larger than the value that we find for our data.

Here we are dealing with ansätze that are only correct up to corrections that decay with a power of the linear lattice size $L$. As a result, taking into account the smallest $L$ that we have simulated, $\chi^{2} /$ d.o.f. is large and $Q$ very small. Increasing $L_{m i n}$, typically $\chi^{2} /$ d.o.f. decreases and $Q$ increases. In all cases discussed below, eventually acceptable values of $\chi^{2} /$ d.o.f. and $Q$ are reached. In our plots below we give only estimates that correspond to $Q>0.01$. Typically $Q$ rapidly increases going to slightly larger $L_{\min }$. For most of the estimates shown $Q>0.1$. A large value of $\chi^{2} /$ d.o.f. or a small value of $Q$ certainly indicates that the ansatz that is used is not sufficient to describe the data. Unfortunately, however an acceptable value of $\chi^{2} /$ d.o.f. or $Q$ says little about the systematic error on the parameters such as the exponent $u$. In particular the systematic error can be considerably larger than the statistical one that is provided by the fit. This can be seen explicitly for example in our data for the slopes of different phenomenological couplings. While the correction exponents 
are the same for different quantities, very likely the corresponding amplitudes are not. Hence the systematic effect on, for example, the result for the exponent $y_{t}$ is likely different for different quantities. And in fact we see differences in $y_{t}$ obtained from different quantities that are clearly larger than the statistical error, despite the fact that $Q$ is acceptable. This effect can also be easily seen by generating synthetic data according to a function $g$ with given values of the parameters and then fitting by using the ansatz $f$, where $f$ is obtained from $g$ by skipping correction terms.

In order to get some handle on the systematic error we compare results obtained by the same ansatz but different quantities or by different ansätze, containing a different number of correction terms for the same quantity. The final analysis is performed graphically. We plot the estimate of, for example, $y_{t}$ as a function of $L_{\text {min }}$. The final result and its error is then chosen such that for all quantities or all ansätze considered the estimate obtained by fitting is, including the respective statistical error, within the interval given by the final estimate plus or minus its error. This procedure is not fully automatized and subject to some judgment.

The least square fits were performed by using the function curve_fit() contained in the SciPy library [31]. The function curve_fit() acts as a wrapper to functions contained in the MINPACK library [32]. We checked the outcome of the fit by varying the initial values of the parameters. Furthermore, we performed fits both by using the Levenberg-Marquardt algorithm and the trust region reflective algorithm. In particular in the case of fits with many free parameters, the trust region reflective algorithm turns out to be more reliable than the Levenberg-Marquardt algorithm. Plots were generated by using the Matplotlib library [33].

\section{THE SIMULATIONS}

Our simulations were performed on various PCs and servers. The CPU times quoted below refer to a single core of an $\operatorname{Intel}(\mathrm{R}) \mathrm{Xeon}(\mathrm{R}) \mathrm{CPU}$ E3-1225 v3 running at $3.20 \mathrm{GHz}$, which is the CPU of our PC at home. For example for an AMD EPYC ${ }^{T M} 7351 \mathrm{P}$ CPU we find very similar times, running the program on a single core.

First we performed a number of preliminary simulations to map out the phase diagram of the model. There is a line of second-order phase transitions that starts at $D=\infty$ extending 
to $D_{\text {tri }} \approx-0.5$. For smaller values of $D$, the transition is of first order. Our preliminary estimate for the improved model is $D^{*} \approx 2$. We also obtained preliminary estimates of the inverse critical temperature $\beta_{c}(D)$ for various values of $D$. Based on these preliminary results we arranged our main simulations.

For $D=2.05$ and 2.1 we simulated the linear lattice sizes $L=4,5, \ldots, 14,16, \ldots, 24$, $28, \ldots, 48,56, \ldots, 80,90,100,140,200$, and 400 . In the case of $D=2.0$ we simulated the same lattice sizes up to $L=64$. Larger lattice sizes are only $L=80$ and 200. For example for $D=2.1$ we performed about $3 \times 10^{9}$ measurements up to $L=32$. Then the statistics is slowly decreasing to $6.6 \times 10^{8}$ measurements for $L=100$. We performed $3.5 \times 10^{8}$, $1.45 \times 10^{8}$, and $1.8 \times 10^{7}$ measurements for $L=140,200$, and 400 . Most of the simulations were performed by using cycle A. For $L=90$ and 400, cycle B was used.

The simulations for $D=2.0,2.05$ and 2.1 took in total 60 years of CPU time. These simulations were performed to accurately determine the fixed point values $R_{i}^{*}$ of dimensionless quantities and $D^{*}$. The critical exponents $\nu$ and $\eta$ are determined by using data generated for $D=2.05$ and 2.1 .

In addition we simulated at $D=\infty, 1.4,1.0,0.5,0.0$, and -0.3 using lattice sizes up to $L=90$. This set of simulations mainly serves to determine the exponent of leading corrections to scaling $\omega$. Furthermore improved observables are constructed based on these data. Also for these simulations, we spent in total 60 years of CPU time.

\section{A. Fixed point values of the RG-invariant quantities and critical temperatures}

In this section, we determine the critical temperature for $D=2.0,2.05$, and $2.1, D^{*}$ and the fixed point values of phenomenological couplings. First we analyze the phenomenological couplings one by one, similar to the analysis performed in section $\mathrm{V}$ A of ref. [7]. We use the ansätze

$$
\begin{aligned}
& R_{i}\left(L, D, \beta_{c}(D)\right)=R_{i}^{*}, \\
& R_{i}\left(L, D, \beta_{c}(D)\right)=R_{i}^{*}+b_{i}(D) L^{-\epsilon_{1}}, \\
& R_{i}\left(L, D, \beta_{c}(D)\right)=R_{i}^{*}+b_{i}(D) L^{-\epsilon_{1}}+c_{i}(D) L^{-\epsilon_{2}}, \\
& R_{i}\left(L, D, \beta_{c}(D)\right)=R_{i}^{*}+b_{i}(D) L^{-\epsilon_{1}}+c_{i}(D) L^{-\epsilon_{2}}+d_{i}(D) L^{-\epsilon_{3}},
\end{aligned}
$$


where we approximate

$$
b_{i}(D)=b_{s, i}\left(D-D^{*}\right)
$$

linearly and $c_{i}(D)$ and $d_{i}(D)$ being constant for $D=2.0,2.05$ and 2.1 that we consider here. Note that writing the leading correction amplitude $b_{i}(D)$ this way, $D^{*}$ is an immediate parameter of the fit. Also the slopes $b_{s, i}$ contain important information as we shall see below, eq. (18). We take $\epsilon_{1}=0.76, \epsilon_{2}=2$, and either $\epsilon_{3}=2.2$ or $\epsilon_{3}=4$. Note that $\epsilon_{1}$ is close to the estimate $\omega=0.759(2)$ obtained below. The analytic background of the magnetic susceptibility and the violation of the rotational invariance are effectively taken into account by the term $c L^{-\epsilon_{2}}$. The choice $\epsilon_{3}=2.2$ is motivated by a preliminary estimate of $\omega_{i c o}$. We checked that taking for example $\epsilon_{3}=2.17$ instead, changes the estimates of the critical temperature and $R_{i}^{*}$ by little. Adding a term $c L^{-4}$ is mainly driven by the observation that this way $\chi^{2} /$ d.o.f. $\approx 1$ are obtained down to $L_{\min }=5$. This observation suggests that there is a correction with an RG-exponent $y \approx-4$ that has a quite large amplitude. Also analyzing different quantities we find that adding a term $c L^{-4}$ results in acceptable fits down to $L_{\min }=5$.

Our final results are mainly based on fits with two correction terms, eq. (15)). Other fits serve to estimate systematic errors. Our results are summarized in table 1 .

In contrast to previous work [7], we made an attempt to jointly fit all four phenomenological couplings $R$ that we consider. To this end we computed the covariances of the different $R$. Since only quantities with the same $D$ and $L$ are correlated, the covariance matrix is sparse. Only four by four blocks are non-vanishing. For example for $L_{\min }=8$, there are $22+27+27$ different $(D, L)$ pairs. Hence the covariance matrix is a $[4(22+27+27)] \times[4(22+27+27)]$ matrix. We passed the full $[4(22+27+27)] \times[4(22+27+27)]$ covariance matrix to optimize.curve_fit, since we found no simple way to indicate that the matrix is sparse. Since the optimization typically took a few seconds, we made no effort to improve on this.

It turns out that in these joint fits, we can include more correction terms. As above, we fixed $\epsilon_{1}=0.76$ corresponding to the exponent of the leading correction $\omega$. We consider the sequence $\epsilon_{i}=2-\eta, 2.02$, and 2.19 of subleading corrections exponents.

We find that $Q>0.1$ for $L_{\min } \geq 22,11$, and 9 , taking into account 1,2 or 3 subleading correction terms, respectively. Here, adding a correction $\propto L^{-4}$ does not improve the fits much.

In Fig. 1 we give the results obtained for $D^{*}$ by using these fits as a function of the 
TABLE II. In the first column the phenomenological coupling is specified. In the second column we give the corresponding estimates of the fixed point values $R^{*}$ obtained by separate fits for each phenomenological coupling. In the third column we give the estimates of the fixed point values $R^{*}$ obtained by joint fits of all four phenomenological couplings. In the fourth column we give the estimates of $D^{*}$, where leading corrections to scaling vanish. In the following columns, the estimates of the inverse critical temperature $\beta_{c}$ for $D=2.0,2.05$, and 2.1 are given. In rows one to four we give the estimates obtained by fitting the phenomenological coupling separately, while in the last row we give estimates obtained from joint fits.

\begin{tabular}{ccccccc}
\hline$R$ & $R_{\text {sep }}^{*}$ & $R_{\text {joint }}^{*}$ & $D^{*}$ & $\beta_{c}(2.0)$ & $\beta_{c}(2.05)$ & $\beta_{c}(2.1)$ \\
\hline$Z_{a} / Z_{p}$ & $0.19479(6)$ & $0.19477(2)$ & $2.1(1)$ & $0.74542805(10)$ & $0.74296024(7)$ & $0.74060257(7)$ \\
$\xi_{2 n d} / L$ & $0.564005(30)$ & $0.56404(2)$ & $2.14(5)$ & $0.74542795(8)$ & $0.74296021(6)$ & $0.74060251(6)$ \\
$U_{4}$ & $1.13933(4)$ & $1.13929(2)$ & $2.06(3)$ & $0.74542800(9)$ & $0.74296018(8)$ & $0.74060255(8)$ \\
$U_{6}$ & $1.41985(15)$ & $1.41974(5)$ & $2.06(3)$ & $0.74542800(9)$ & $0.74296018(8)$ & $0.74060255(8)$ \\
\hline joint & & & $2.08(2)$ & $0.74542801(5)$ & $0.74296024(5)$ & $0.74060256(5)$ \\
\hline
\end{tabular}

minimal lattice size $L_{\text {min }}$. In the plot, only results that correspond to $Q>0.01$ are given. The final estimate of $D^{*}$ and its error bar are chosen such that the estimates of $D^{*}$ obtained by the individual fits, including their respective error bars, are contained in the interval that is given by the final estimate plus or minus its error for some range of $L_{m i n}$. For example, the results for the ansatz only containing the subleading correction term $\propto L^{-2+\eta}$ are within this interval up to $L_{\min }=40$.

In a similar fashion we determine the final estimates of the fixed point values of the phenomenological couplings and the inverse critical temperatures. These are summarized in table 【I.

1. Eliminating leading corrections to scaling in dimensionless quantities

We construct linear combinations of two phenomenological couplings

$$
R_{i m p, i, j}=R_{i}+p_{i, j} R_{j}
$$




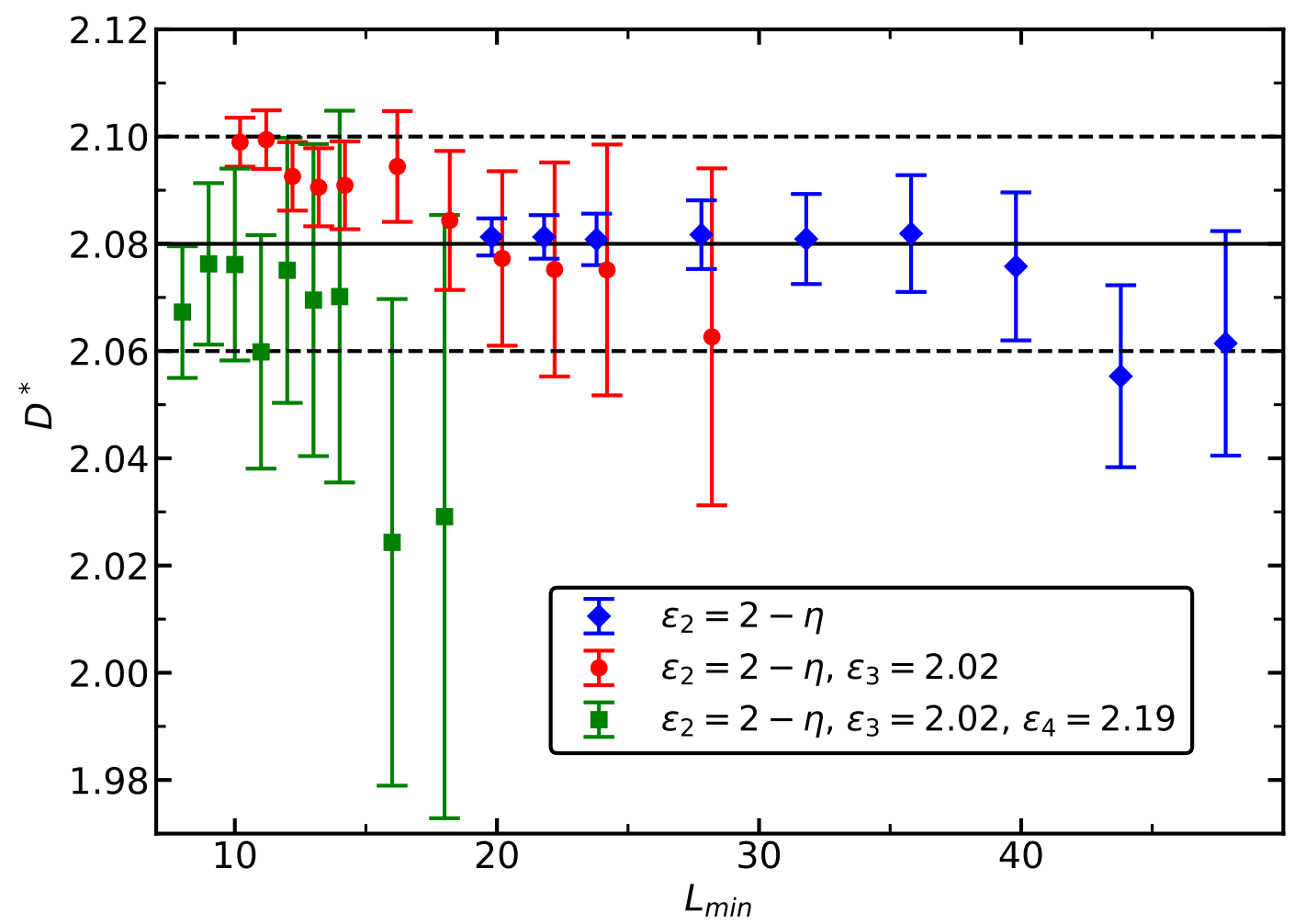

FIG. 1. We plot the estimate of $D^{*}$ obtained by using joint fits of phenomenological couplings as function of the minimal lattice size $L_{\min }$ that is taken into account. Data for $D=2.0,2.05$ and 2.1 are used in the fits. Only results for $Q>0.01$ are given. To make the figure more readable, we have shifted the values of $L_{\min }$ slightly. The solid line indicates the preliminary estimate based on this set of fits. The dashed lines give the error estimate. The exponents $\epsilon_{i}$ given in the legend refer to the correction terms that are included in the ansätze.

such that leading corrections to scaling are eliminated. To this end, we make use of the parameter $b_{s, i}$, eq. (17), which we determined in the analysis of the phenomenological couplings discussed above. One gets

$$
p_{i, j}=-\frac{b_{s, i}}{b_{s, j}}
$$

Note that these results hold for any model in the three-dimensional Heisenberg universality class. Here we consider the combination of either $Z_{a} / Z_{p}$ or $\xi_{2 n d} / L$ with $U_{4}$. Our numerical estimates are summarized in table III.

Jointly fitting the data for $D=2.0,2.05$ and 2.1, assuming that leading corrections to scaling vanish, we find $\left(Z_{a} / Z_{p}+0.575 U_{4}\right)^{*}=0.84987(3)$ and $\left(\xi_{2 n d} / L-0.75 U_{4}\right)^{*}=$ 
TABLE III. Estimates of the coefficient $p_{i, j}$, eq. (18), needed to construct improved phenomenological couplings.

\begin{tabular}{ccc}
\hline$R_{i}$ & $R_{j}$ & $p_{i, j}$ \\
\hline$Z_{a} / Z_{p}$ & $U_{4}$ & $0.575(25)$ \\
$\xi_{2 n d} / L$ & $U_{4}$ & $-0.750(25)$ \\
\hline
\end{tabular}

TABLE IV. Estimates of $\beta_{c}$ for the values of $D$ different from $D=2.0,2.05,2.1$.

\begin{tabular}{cc}
\hline$D$ & $\beta_{c}$ \\
\hline$\infty$ & $0.6925051(2)$ \\
1.4 & $0.7854535(2)$ \\
1.0 & $0.8260052(2)$ \\
0.5 & $0.8979286(2)$ \\
0.0 & $0.9986988(2)$ \\
-0.3 & $1.0742253(4)$ \\
\hline
\end{tabular}

$-0.290437(10)$. Note that these results are consistent with those obtained by naively combining the estimates of $R^{*}$ given in table II] Since the leading correction to scaling is eliminated up to the numerical uncertainty of the coefficients $p_{i j}$, these linear combinations are well suited to determine the inverse critical temperature $\beta_{c}$ of models that are not improved. Furthermore, in the slope of these combinations the effective correction $\propto L^{-y_{t}-\omega}$ is eliminated. In the analysis of the generalized icosahedral model we shall not make use of this fact, since $\omega_{i c o}$ assumes a value that is similar to $y_{t}+\omega$.

In table IV we give estimates of $\beta_{c}$ obtained by analyzing $Z_{a} / Z_{p}+0.575 U_{4}$. We use the estimate of $\left(Z_{a} / Z_{p}+0.575 U_{4}\right)^{*}$ given above as input. The error quoted also takes into account the uncertainty of $\left(Z_{a} / Z_{p}+0.575 U_{4}\right)^{*}$.

Note that the value of $\beta_{c}$ for $D=\infty$ is slightly smaller than $\beta_{c}=0.693002(2)$ for the $O(3)$-invariant Heisenberg model on the simple cubic lattice [34]. 


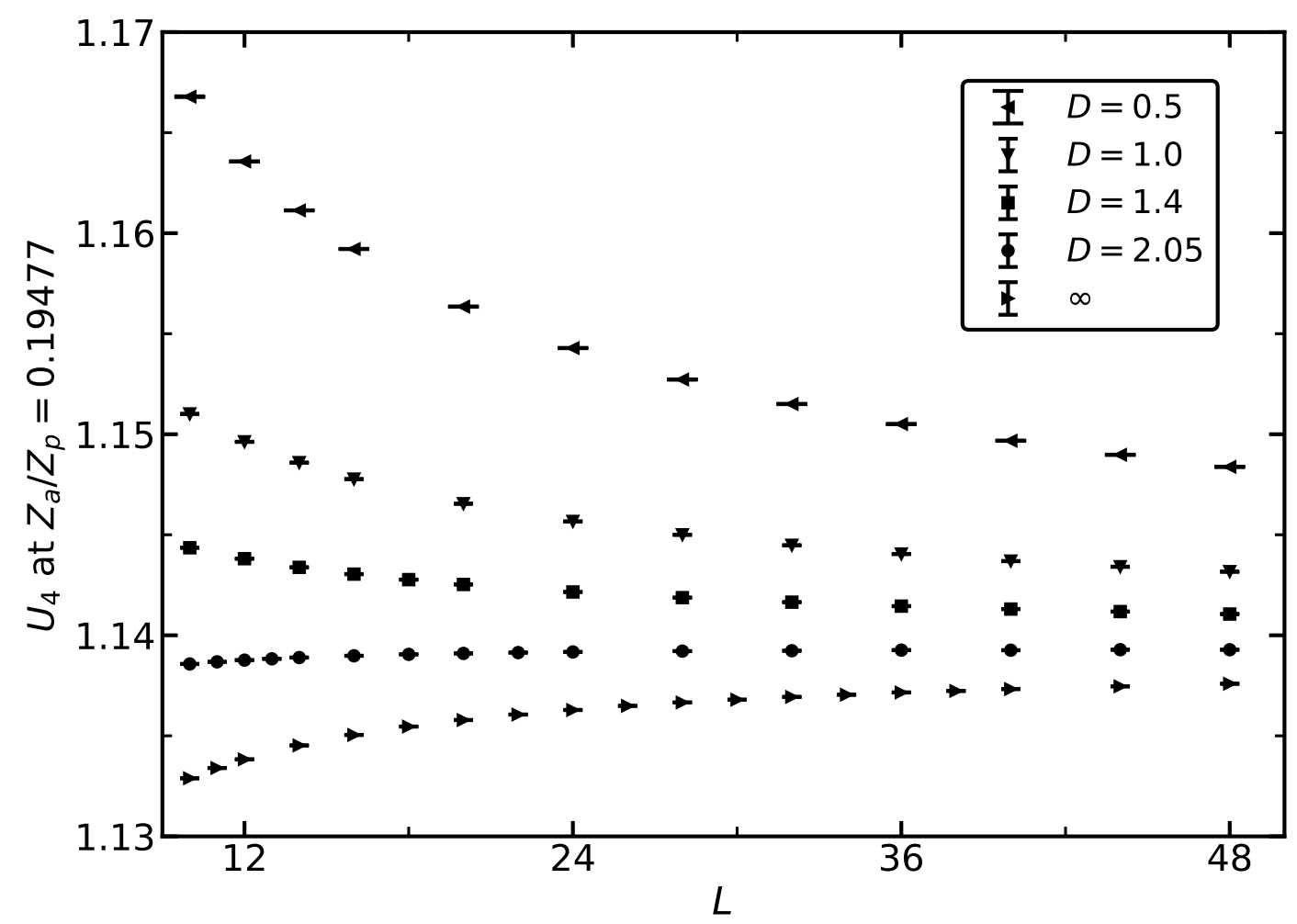

FIG. 2. We plot $U_{4}$ at $Z_{a} / Z_{p}=0.19477$ for $D=0.5,1.0,1.4, D=2.05$ and $\infty$ as a function of the linear lattice size $L$.

\section{B. Leading corrections to scaling}

In this section we focus on leading corrections to scaling. To this end we consider the cumulants $U_{4}$ and $U_{6}$ at $Z_{a} / Z_{p}=0.19477$ or $\xi_{2 n d} / L=0.56404$, which are our estimates of the fixed point values of these quantities. This means that $U_{4}$ and $U_{6}$ are taken at $\beta_{f}$, where $\beta_{f}$ is chosen such that either $Z_{a} / Z_{p}=0.19477$ or $\xi_{2 n d} / L=0.56404$. In the following we denote a cumulant at a fixed value of $Z_{a} / Z_{p}$ or $\xi_{2 n d} / L$ by $\bar{U}$.

To get a first impression, we plot in Fig. 2 the Binder cumulant $U_{4}$ at $Z_{a} / Z_{p}=0.19477$ for $D=0.5,1.0,1.4,2.05$ and $\infty$. To keep the figure readable, we do not plot the data for $D=2.0$ and 2.1, which are similar to those of $D=2.05$. For $D=1.4$ the correction amplitude has roughly the same modulus as for $D=\infty$, but opposite sign. The amplitude of leading corrections increases with decreasing $D$. The analysis performed below shows that the amplitudes of leading corrections for $D=0.0$ and -0.3 are about 2.7 and 7 times as large as for $D=0.5$, respectively. The results obtained for $\xi_{2 n d} / L=0.56404$ are similar. 
The results obtained for $U_{6}$ are qualitatively the same as for $U_{4}$.

We performed joint fits for several sets of $D$. Either all values of $D$ are taken into account, or subsets of them. These subsets are obtained by skipping values of $D$ starting from the smallest one. The minimal set that we consider consists of $D=1.4,2.0,2.05,2.1$, and $\infty$. Similar to ref. [7] we analyzed our data by using the ansatz

$$
\bar{U}=\bar{U}^{*}+\sum_{i=1}^{i_{\max }} c_{i}\left[b(D) L^{-\omega}\right]^{i}+d L^{-\epsilon}
$$

for various values of $i_{\max }$. In order to avoid ambiguity, we set $c_{1}=1$. The free parameters of the fit are $\bar{U}^{*}, c_{2}, c_{3}, \ldots, b(D)$ for each value of $D$. In our fits, the parameter $d$ is the same for all values of $D$. In our fits we set $\epsilon=2$.

A preliminary study shows that the results obtained for $\xi_{2 n d} / L=0.56404$ are more stable than those for $Z_{a} / Z_{p}=0.19477$. In particular, the values of $c_{2}, c_{3}, \ldots$ have a smaller modulus for $\xi_{2 n d} / L=0.56404$ than for $Z_{a} / Z_{p}=0.19477$. Therefore in the following we shall focus on $U_{4}$ and $U_{6}$ at $\xi_{2 n d} / L=0.56404$.

In Fig. 3 we plot our results for the correction exponent $\omega$ obtained from joint fits of $U_{4}$ at $\xi_{2 n d} / L=0.56404$ for $D=0.5, \ldots, \infty$. We give our results for $i_{\max }=1,2$ and 3 as a function of the minimal lattice size $L_{\text {min }}$ that is taken into account. We find that fits with $i_{\max }=2$ and 3 are consistent and acceptable fits are obtained starting from $L_{\min }=12$. In contrast, for $i_{\max }=1$ we get $Q>0.1$ only for $L_{\min } \geq 40$. The estimate of $\omega$ obtained with $i_{\max }=1$ is considerably smaller than for $i_{\max }=2$ and 3 . As our preliminary estimate we take $\omega=0.7589(10)$ from $L_{\text {min }}=20$.

Performing a similar analysis, taking into account all values of $D$, we get consistent results for $\omega$ starting from $i_{\max }=3$ and 4 . Here we take $\omega=0.7595(10)$ from $L_{\min }=24$ as preliminary estimate. Taking the set $D=1.4, \ldots, \infty$ we get already consistent results for $\omega$ with $i_{\max }=1$ and 2 . Here we take $\omega=0.7584(10)$ from $L_{\min }=20$ as preliminary estimate. As a check we performed fits without the term $d L^{-\epsilon}$. We find that $\chi^{2} /$ d.o.f. considerably increases. However the estimates for $\omega$ are consistent with those obtained from fits with such a term.

Analyzing $U_{6}$ at $\xi_{2 n d} / L=0.56404$ we get similar results as for $U_{4}$.

As our final estimate we quote

$$
\omega=0.759(2)
$$




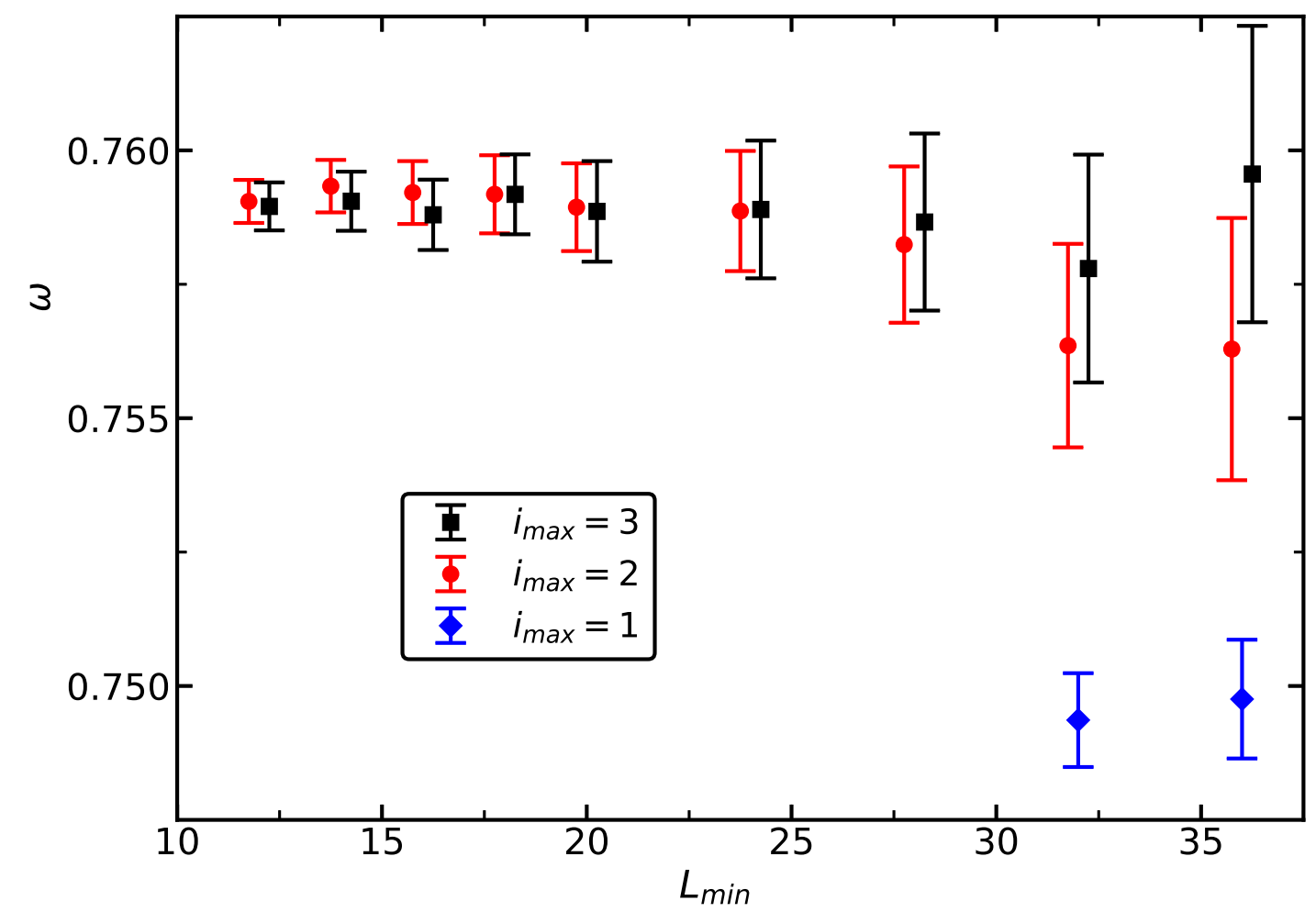

FIG. 3. We plot the estimates of $\omega$ obtained by fitting $U_{4}$ at $\xi_{2 n d} / L=0.56404$ for $D=0.5,1.0$, $1.4,2.0,2.05,2.1$, and $\infty$ by using the ansatz (20) as a function of the minimal lattice size $L_{\min }$ that is taken into account. Only results for $Q>0.01$ are given. To make the figure more readable, we have shifted the values of $L_{\min }$ slightly.

which covers the preliminary estimates discussed above. From the analysis of $U_{4}$ and $U_{6}$ at $Z_{a} / Z_{p}=0.19477$ we would arrive at $\omega=0.758(4)$. Since the fits for $\xi_{2 n d} / L=0.56404$ are clearly better behaved than those for $Z_{a} / Z_{p}=0.19477$, we stick with the result obtained from $\xi_{2 n d} / L=0.56404$ as our final estimate.

C. $D^{*}$

Here we analyze $\bar{U}_{4}$ and $\bar{U}_{6}$ at $D=2.0,2.05$ and 2.1. To this end we consider the ansätze

$$
\begin{aligned}
& \bar{U}=\bar{U}^{*}+b(D) L^{-\epsilon_{1}}+c L^{-\epsilon_{2}}, \\
& \bar{U}=\bar{U}^{*}+b(D) L^{-\epsilon_{1}}+c L^{-\epsilon_{2}}+d L^{-\epsilon_{3}},
\end{aligned}
$$




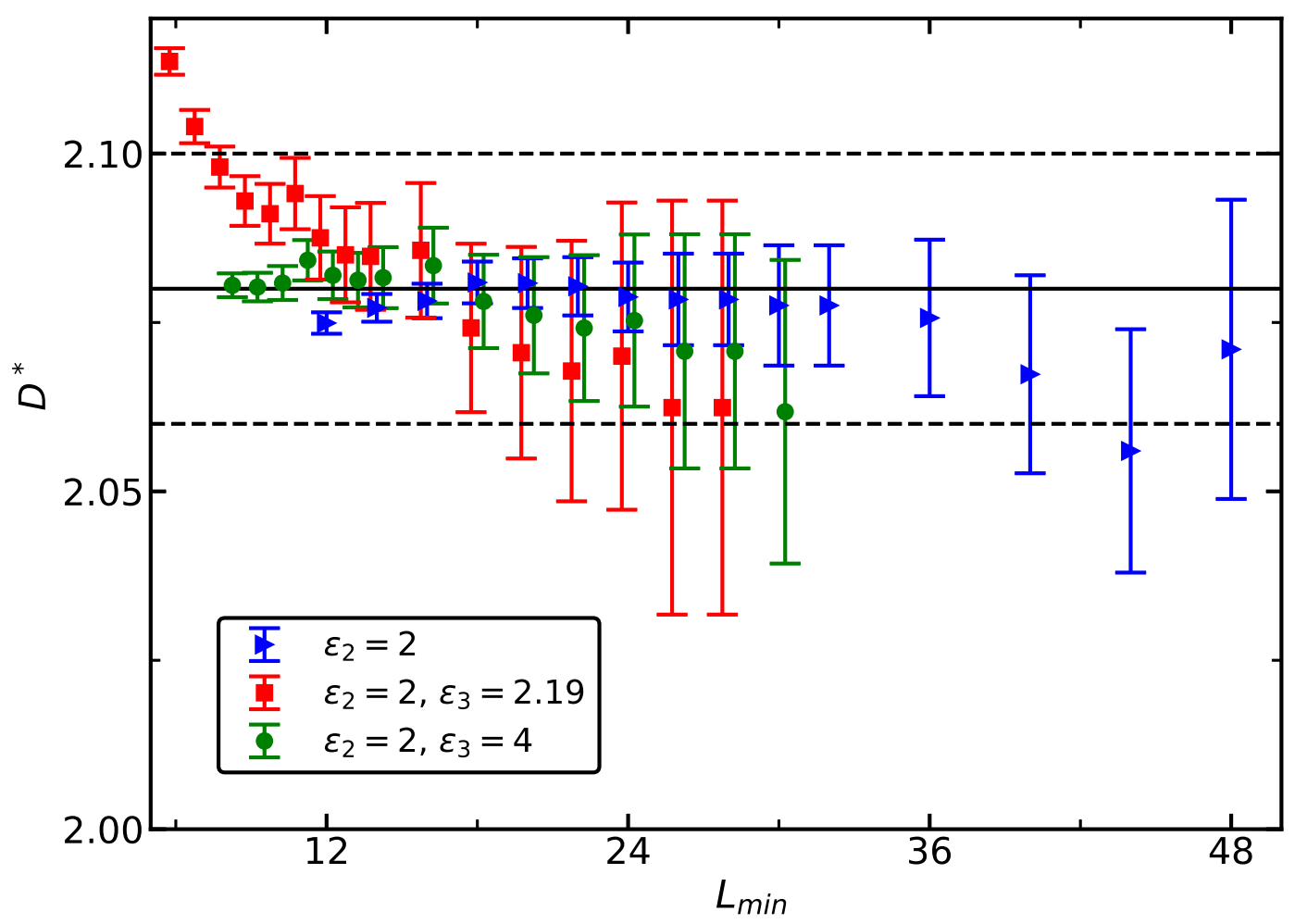

FIG. 4. We plot estimates of $D^{*}$ obtained from fitting $U_{4}$ at $\xi_{2 n d} / L=0.56404$ by using the ansätze (2223) as a function of $L_{\text {min }}$. Only results for $Q>0.01$ are given. To make the figure more readable, we have shifted the values of $L_{\text {min }}$ slightly. The solid line indicates the preliminary results based on this set of fits. The dashed lines give the error estimate.

where we fix $\epsilon_{1}=0.759$, which is our estimate of $\omega$ obtained above, $\epsilon_{2}=2$, which effectively takes into account $2-\eta$ and $\omega_{N R}$. We take either $\epsilon_{3}=2.19$, which corresponds to $\omega_{i c o}$, or $\epsilon_{3}=4$. We parameterize the leading correction as $b(D)=b_{s}\left(D-D^{*}\right)$, where $b_{s}$ and $D^{*}$ are free parameters of the fit. Furthermore $\bar{U}^{*}, c$ and $d$ are free parameters. Here we assume that $c$ and $d$ are the same for all three values of $D$, which should be a reasonable approximation. Below we focus on $\bar{U}_{4}$, since the results for $\bar{U}_{6}$ are similar. In Fig. 4 we plot results obtained for $D^{*}$ by fitting $U_{4}$ at $\xi_{2 n d} / L=0.56404$ using the ansätze (22),23). As our preliminary estimate we take $D^{*}=2.08(2)$. In a similar fashion we arrive at $\bar{U}_{4}^{*}=1.139295(20)$ for $\xi_{2 n d} / L=0.56404$.

Analyzing $U_{4}$ at $Z_{a} / Z_{p}=0.19477$ we arrive at $D^{*}=2.07(3)$ and $\bar{U}_{4}^{*}=1.13930(2)$. 
As our final estimate for the improved model we take

$$
D^{*}=2.08(2)
$$

which is the result of the joint analysis performed in section IVA and the analysis of $U_{4}$ at $\xi_{2 n d} / L=0.56404$.

\section{The critical exponent $\nu$ of the correlation length}

We compute the exponent $\nu=1 / y_{t}$ from the slope of a phenomenological coupling $R_{j}$ at a given value $R_{i, f}$ of a second quantity $R_{i}$, where $R_{j}$ and $R_{i}$ might be the same. Following the discussion of section III C of ref. [7] these slopes behave as

$$
\begin{aligned}
\bar{S}_{i, j}=\left.\frac{\partial R_{j}}{\partial \beta}\right|_{R_{i}=R_{i, f}} & =a L^{y t}\left[1+b L^{-\omega}+\ldots+c_{b a c k} L^{-2-\eta}+c_{N R} L^{-\omega_{N R}}+c_{i c o} L^{-\omega_{i c o}}+\ldots\right] \\
& +d L^{-\omega}+\ldots
\end{aligned}
$$

Note that the coefficients $a, b, c_{b a c k}, c_{N R}, c_{i c o}$ and $d$ depend on the quantity that is considered and on the model, which means in the present case on the parameter $D$. As discussed in ref. [7] and references therein it is advantageous to take $R_{i, f} \approx R_{i}^{*}$, since otherwise an effective correction $\propto\left(R_{i, f}-R_{i}^{*}\right) L^{-y_{t}}$ has to be taken into account.

Below we consider $D=2.05$ and 2.1 which are close to $D^{*}$. Therefore the coefficient $b$ of the leading correction is small for all quantities. In order to ensure that leading corrections to scaling can be safely ignored at the level of our accuracy, we construct improved slopes by multiplying $\bar{S}$ by a certain power $p$ of the Binder cumulant $\bar{U}_{4}$ :

$$
\bar{S}_{i m p}=\bar{S} \bar{U}_{4}^{p}
$$

where both $\bar{S}$ and $\bar{U}_{4}$ are taken at $R_{i, f}$. The exponent $p$ is chosen such that, at the level of our numerical accuracy, leading corrections to scaling are eliminated. This idea is discussed systematically in ref. [35]. To determine $p$, we consider the pair $\left(D_{1}, D_{2}\right)=(1.4, \infty)$. Note that as discussed in section IVB, the amplitude of the leading correction to scaling for these two values of $D$ has approximately the same modulus but opposite sign. We fit ratios of $\bar{S}_{i, j}$ and $\bar{U}_{4}$ with the ansätze

$$
\frac{\bar{S}_{i, j}\left(D_{1}\right)}{\bar{S}_{i, j}\left(D_{2}\right)}=a_{S}\left(1+b_{S} L^{-\epsilon_{1}}\right), \quad \frac{\bar{S}_{i, j}\left(D_{1}\right)}{\bar{S}_{i, j}\left(D_{2}\right)}=a_{S}\left(1+b_{S} L^{-\epsilon_{1}}+c_{S} L^{-\epsilon_{2}}\right)
$$


TABLE V. Numerical result for the exponent $p$ that eliminates leading corrections to scaling in $\bar{S}_{i j}$, eq. (26).

\begin{tabular}{ccccc}
\hline Fixing $\backslash$ Slope of & $Z_{a} / Z_{p}$ & $\xi_{2 n d} / L$ & $U_{4}$ & $U_{6}$ \\
\hline$Z_{a} / Z_{p}=0.19477:$ & $1.65(10)$ & $0.24(10)$ & $-3.6(2)$ & $-5.0(3)$ \\
$\xi_{2 n d} / L=0.56404:$ & $0.07(7)$ & $0.24(10)$ & $-4.22(10)$ & $-5.64(10)$ \\
\hline
\end{tabular}

and

$$
\frac{\bar{U}_{4}\left(D_{1}\right)}{\bar{U}_{4}\left(D_{2}\right)}=1+b_{U} L^{-\epsilon_{1}}, \quad \frac{\bar{U}_{4}\left(D_{1}\right)}{\bar{U}_{4}\left(D_{2}\right)}=1+b_{U} L^{-\epsilon_{1}}+c_{U} L^{-\epsilon_{2}},
$$

where we fixed $\epsilon_{1}=0.76$ and $\epsilon_{2}=2$. The exponent $p$ is given by

$$
p=-\frac{b_{S}}{b_{U}}
$$

In table $\nabla$ we give our final results for $p$. The error bar takes into account statistical errors as well as systematical ones, which are estimated by comparing the results of the two different ansätze.

Let us briefly comment on the statistical error of the different quantities. We find that both fixing $Z_{a} / Z_{p}=0.19477$ and $\xi_{2 n d} / L=0.56404$ changes, compared with fixed $\beta$, the relative statistical error of the slopes only little. The same holds for the comparison of the improved and the unimproved slopes. We see big differences between the relative statistical errors of the slopes of the different phenomenological couplings. The relative error is the smallest in the case of $\xi_{2 n d} / L$. The ratios of the statistical errors vary only little with the linear lattice size. For example for $D=2.05$ and $L=40$ at $\beta_{c}$ we find that the relative statistical error of the slope of $Z_{a} / Z_{p}, U_{4}$ and $U_{6}$ is by a factor of $1.24,1.99$, and 2.00 larger than that of $\xi_{2 n d} / L$. As a measure of the effort to reach a certain accuracy, beyond the increase due to the increasing lattice size, we studied $w=n_{\text {stat }} \epsilon_{r}^{2}$, where $n_{\text {stat }}$ is the number of measurements and $\epsilon_{r}$ the relative statistical error. We fitted the data for the slope of $Z_{a} / Z_{p}$ at $Z_{a} / Z_{p}=0.19477$ for $D=2.05$. We find a behavior $w \propto L^{x}$ with $x \approx 0.36$. Since we already averaged over bins during the simulation, we can not determine to what extend this degradation of the efficiency is due to an increase of autocorrelation times or and increase of the variance of the slope.

Below we perform throughout joint fits of the data for $D=2.05$ and $D=2.1$. In these 
fits, the overall amplitude for each value of $D$ is a free parameter of the fit. In contrast, we assume that the correction amplitudes are similar, and are taken to be same in the ansatz.

First we have analyzed the improved slopes of the different phenomenological couplings separately. We have fitted these quantities by using the ansätze

$$
\begin{aligned}
& S=a L^{y_{t}}, \\
& S=a L^{y_{t}}\left(1+b L^{-\epsilon_{1}}\right),
\end{aligned}
$$

where we take $\epsilon_{1}=2$, which should effectively take into account corrections due to the analytic background of the magnetic susceptibility and the violation of the rotational invariance by the simple cubic lattice. First we analyzed our data by using ansatz (30) without correction term. In Fig. [5 we give our results for improved slopes at $\xi_{2 n d} / L=0.56404$. We do not give results for $U_{6}$, since they are very similar to those for $U_{4}$. Note that for example for $L_{\text {min }}=40$ we get $\chi^{2} /$ d.o.f. $=0.949$ and 1.150 for the slopes of $\xi_{2 n d} / L$ and $Z_{a} / Z_{p}$, respectively. This corresponds to $Q=0.526$ and 0.286 , respectively. Despite this fact, the estimates of $y_{t}$ obtained for $L_{\min }=40$ clearly differ for $\xi_{2 n d} / L$ and $Z_{a} / Z_{p}$. As our preliminary estimate we take $y_{t}=1.40520(32)$. It is chosen such that all three results for $L_{m i n}=72$ are covered. The estimates obtained for $Z_{a} / Z_{p}=0.19477$ are similar.

In Fig. 6] we give results obtained from fitting the improved slopes of $Z_{a} / Z_{p}, \xi_{2 n d} / L$ and $U_{4}$ at $\xi_{2 n d} / L=0.56404$ by using the ansatz (31). As our preliminary estimate of this set of fits we take $y_{t}=1.40520(20)$. It covers all three estimates obtained for $L_{\min }=24$. Analyzing the slopes at $Z_{a} / Z_{p}=0.19477$ in a similar way, we find consistent results.

Finally we performed a joint analysis of the improved slopes of all four phenomenological couplings at either $\xi_{2 n d} / L=0.56404$ or $Z_{a} / Z_{p}=0.19477$. Similar to section IVA, we took the covariances of the different quantities into account. In these fits, we used ansätze with up to three different correction terms with the effective correction exponents $\epsilon_{1}=2-\eta$, $\epsilon_{2}=2.02$, and $\epsilon_{3}=2.19$. The first is motivated by the analytic background of the magnetic susceptibility, the second by $\omega_{N R}$ and the third by $\omega_{i c o}$. Note that in the slope we also expect corrections with the exponent $y_{t}+\omega$, which is effectively taken into account by $\epsilon_{3}$. In Fig. 7 we give our results for the improved slopes at $\xi_{2 n d} / L=0.56404$. In Fig. 8 we give the corresponding results for $Z_{a} / Z_{p}=0.19477$. As our preliminary estimates we take 


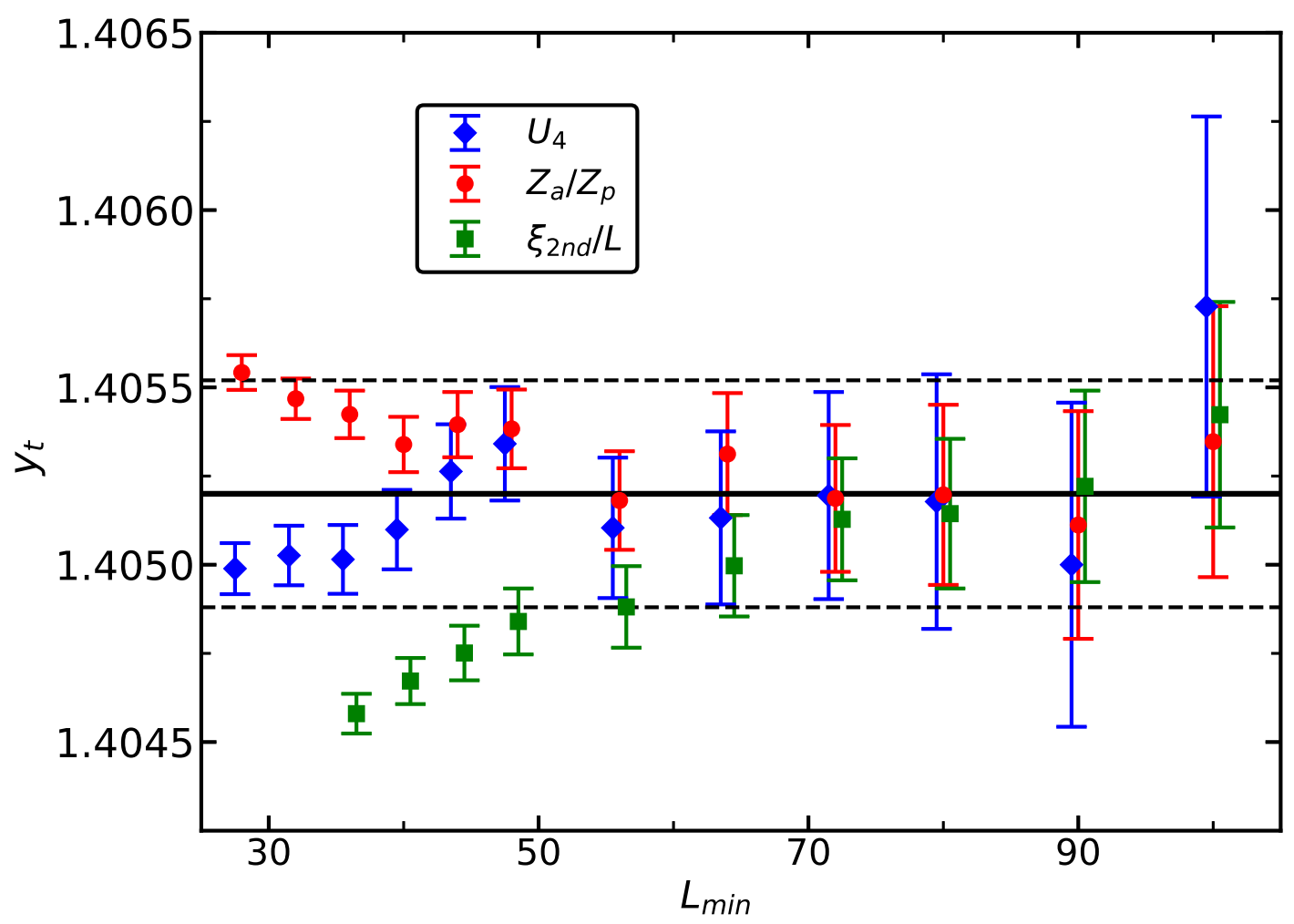

FIG. 5. We plot the estimates of $y_{t}$ obtained from fitting the improved slopes of $Z_{a} / Z_{p}, \xi_{2 n d} / L$ and $U_{4}$ at $\xi_{2 n d} / L=0.56404$ for $D=2.05$ and 2.1 by using the ansatz (30) as a function of the minimal lattice size $L_{\text {min }}$ that is taken into account. Only results for $Q>0.01$ are given. To make the figure more readable, we have shifted the values of $L_{\min }$ slightly. The solid line indicates the preliminary result based on this set of fits. The dashed lines give the error estimate.

$y_{t}=1.40522(18)$ and $1.40525(15)$ obtained for $\xi_{2 n d} / L=0.56404$ and $Z_{a} / Z_{p}=0.19477$, respectively.

Based on these results and the preliminary estimate obtained by fitting the slopes of the different phenomenological couplings separately by using the ansatz (31) we conclude

$$
y_{t}=1.4052(2) \text {, }
$$

which corresponds to $\nu=0.71164(10)$. 


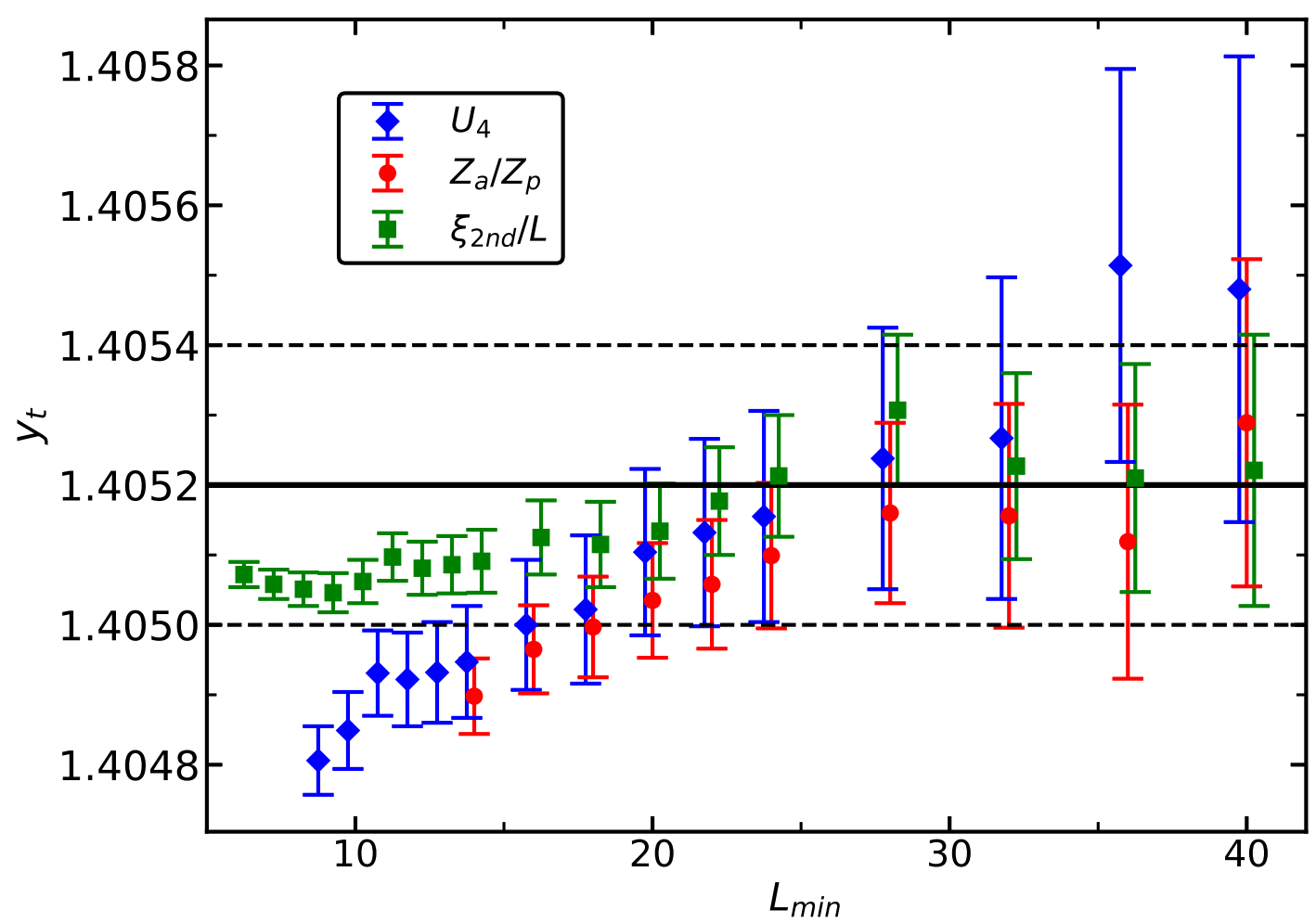

FIG. 6. We plot the estimates of $y_{t}$ obtained from fitting the improved slopes of $Z_{a} / Z_{p}, \xi_{2 n d} / L$ and $U_{4}$ at $\xi_{2 n d} / L=0.56404$ for $D=2.05$ and 2.1 by using the ansatz (31) as a function of the minimal lattice size $L_{\min }$ that is taken into account. Only results for $Q>0.01$ are shown. To make the figure more readable, we have shifted the values of $L_{\min }$ slightly. The solid line indicates our preliminary estimate of this set of fits. The dashed lines give the error estimate.

\section{E. The critical exponent $\eta$}

We analyzed the improved quantities

$$
\bar{\chi}_{i m p}=\bar{\chi} \bar{U}_{4}^{p}
$$

where both $\chi$ and $U_{4}$ are taken either at $Z_{a} / Z_{p}=0.19477$ or $\xi_{2 n d} / L=0.56404$. We computed the exponent $p$ in a similar way as in the previous section for the slopes $S$. Therefore we skip a detailed discussion and only report our results $p=-1.31(3)$ and $-0.23(4)$ for $Z_{a} / Z_{p}=0.19477$ and $\xi_{2 n d} / L=0.56404$, respectively.

Let us briefly discuss the effect of taking $\chi$ at $Z_{a} / Z_{p}=0.19477$ or $\xi_{2 n d} / L=0.56404$ on the statistical error. In previous work, see ref. [7] and references therein, we observed that 


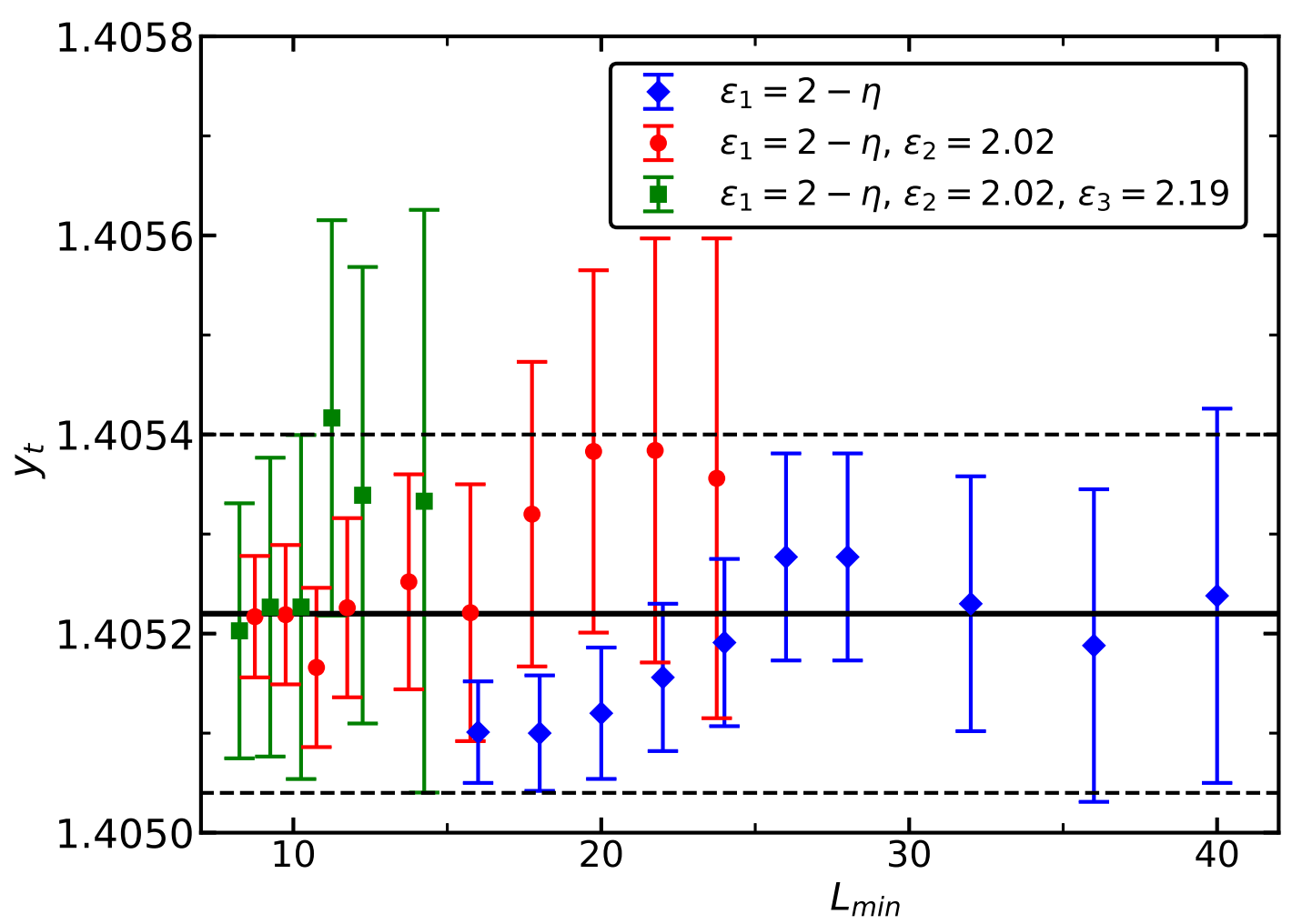

FIG. 7. We plot the estimates of $y_{t}$ obtained by fitting the improved slopes of $Z_{a} / Z_{p}, \xi_{2 n d} / L, U_{4}$ and $U_{6}$ at $\xi_{2 n d} / L=0.56404$ jointly by using up to three different correction terms as a function of the minimal lattice size $L_{\min }$ that is taken into account. Data for $D=2.05$ and $D=2.1$ are taken. Only results for $Q>0.01$ are given. To make the figure more readable, we have shifted the values of $L_{\text {min }}$ slightly. The solid line indicates our preliminary estimate based on this set of fits. The dashed lines give the error estimate. The exponents $\epsilon_{i}$ given in the legend refer to the correction terms that are included in the ansätze.

the statistical error is reduced compared with $\chi$ at a fixed value of $\beta \approx \beta_{c}$. Here we see for $Z_{a} / Z_{p}=0.19477$ only a small effect, while for $\xi_{2 n d} / L=0.56404$ we see for example for $D=2.05$ a reduction of the statistical error by a factor of about two. The relative statistical error of the improved susceptibility is by a few percent larger than that of the unimproved counterpart.

We fitted our data with the ansätze

$$
\begin{aligned}
& \chi=a L^{2-\eta}, \\
& \chi=a L^{2-\eta}+b,
\end{aligned}
$$




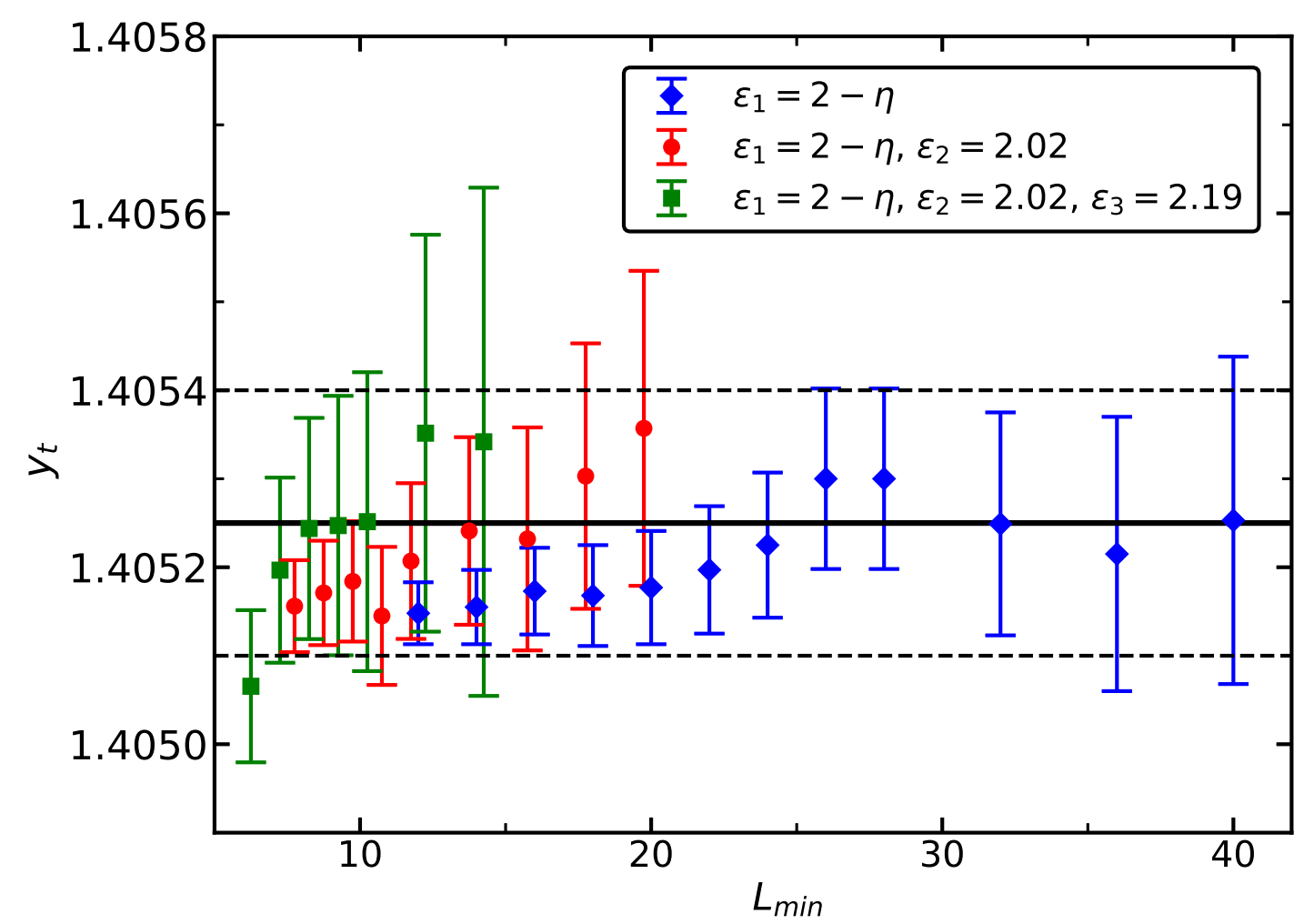

FIG. 8. Same as Fig. 7 but for $Z_{a} / Z_{p}=0.19477$ instead of $\xi_{2 n d} / L=0.56404$.

$$
\chi=a L^{2-\eta}\left(1+c L^{-\epsilon_{2}}\right)+b,
$$

where the analytic background $b$ can be viewed as an effective correction with the exponent $\epsilon_{1}=2-\eta$. Similar to the analysis of the slopes, we performed joint fits of the data for $D=2.05$ and 2.1 .

In Fig. 9 we give the estimates obtained from fitting the improved magnetic susceptibility at $Z_{a} / Z_{p}=0.19477$ by using the ansätze (35,36). In the case of ansatz (36) we plot results for $\epsilon_{2}=2.02$ and 4 . We also performed fits using $\epsilon_{2}=2.19$, which give consistent results for $\eta$. Our preliminary estimate $\eta=0.03784(5)$ for this set of fits is consistent with the estimate obtained by using ansatz (36) with $\epsilon_{2}=2.02$ for $L_{\text {min }}=16$. Furthermore it covers the results obtained by using the ansatz (35) for $L_{\min }=14$ up to 32 and ansatz (36) with $\epsilon_{2}=4$ for $L_{\text {min }} \leq 24$. Fitting the improved magnetic susceptibility at $\xi_{2 n d} / L=0.56404$ by using the ansätze (35, 36) we find results that are consistent with the estimate $\eta=0.03784(5)$.

Finally, in Fig. 10 we plot the estimates obtained from fits of the data for the improved magnetic susceptibility at $\xi_{2 n d} / L=0.56404$ without correction term (34) and with a correc- 


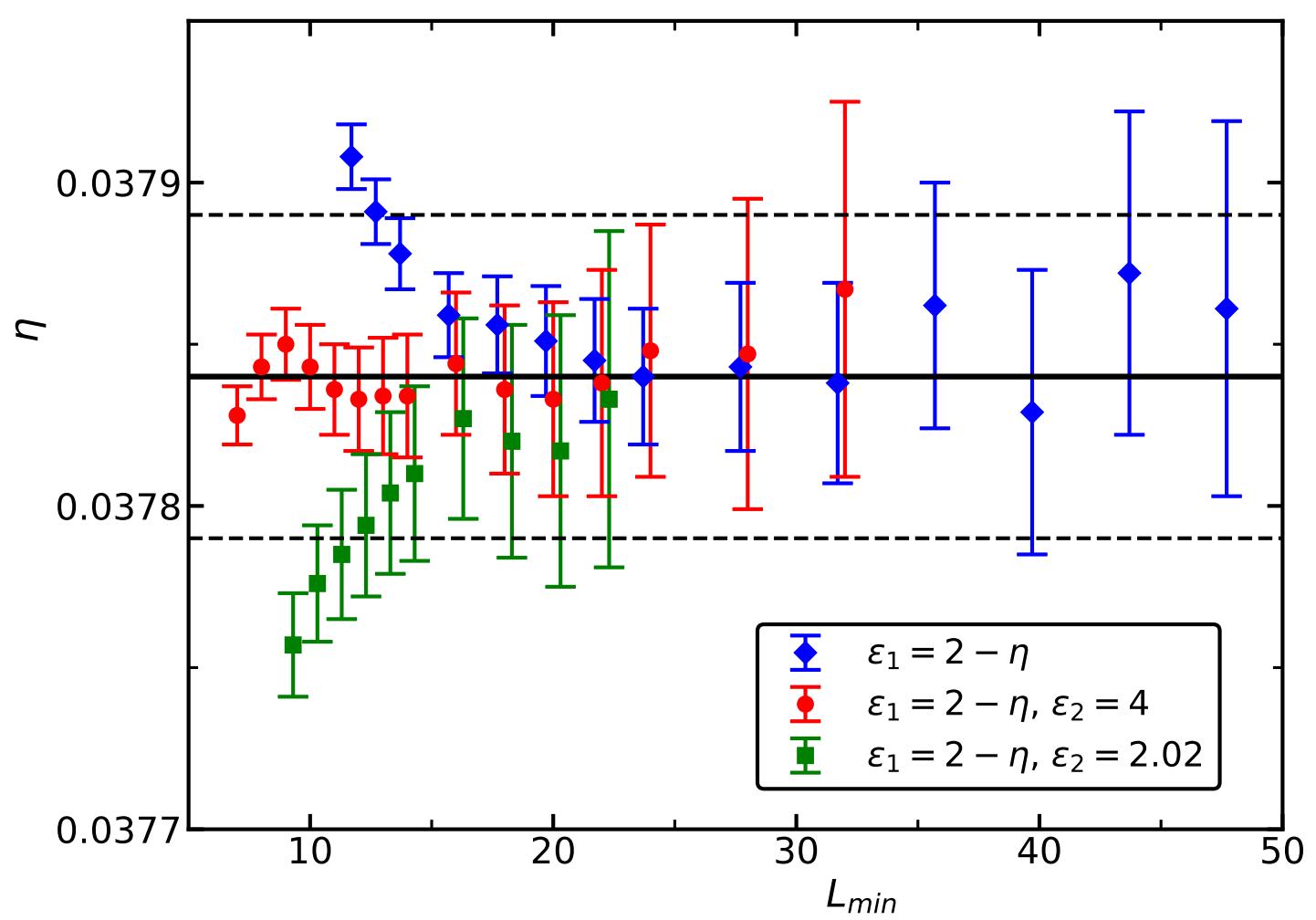

FIG. 9. We plot the estimates of $\eta$ obtained from fitting the improved magnetic susceptibility at $Z_{a} / Z_{p}=0.19477$ by using the ansätze (35/36) as a function of the minimal lattice size $L_{\min }$ that is taken into account. Data for $D=2.05$ and $D=2.1$ are used in the fits. Only results for $Q>0.01$ are given. The numbers given in the legend refer to the corrections that are taken into account in the ansatz. To make the figure more readable, we have shifted the values of $L_{m i n}$ slightly. The solid line gives our preliminary estimate and the dashed lines indicate the error bar.

tion corresponding to the analytic background of the magnetic susceptibility, eq. (35). Based on these fits we arrive at the preliminary estimate $\eta=0.03784(7)$ which takes into account the result obtained by using the ansatz (34) with $L_{\min }=140$ and the results obtained by using the ansatz (35) up to $L_{\min }=80$. As our final estimate of $\eta$ we quote

$$
\eta=0.03784(5)
$$

obtained by using ansätze that include correction terms. 


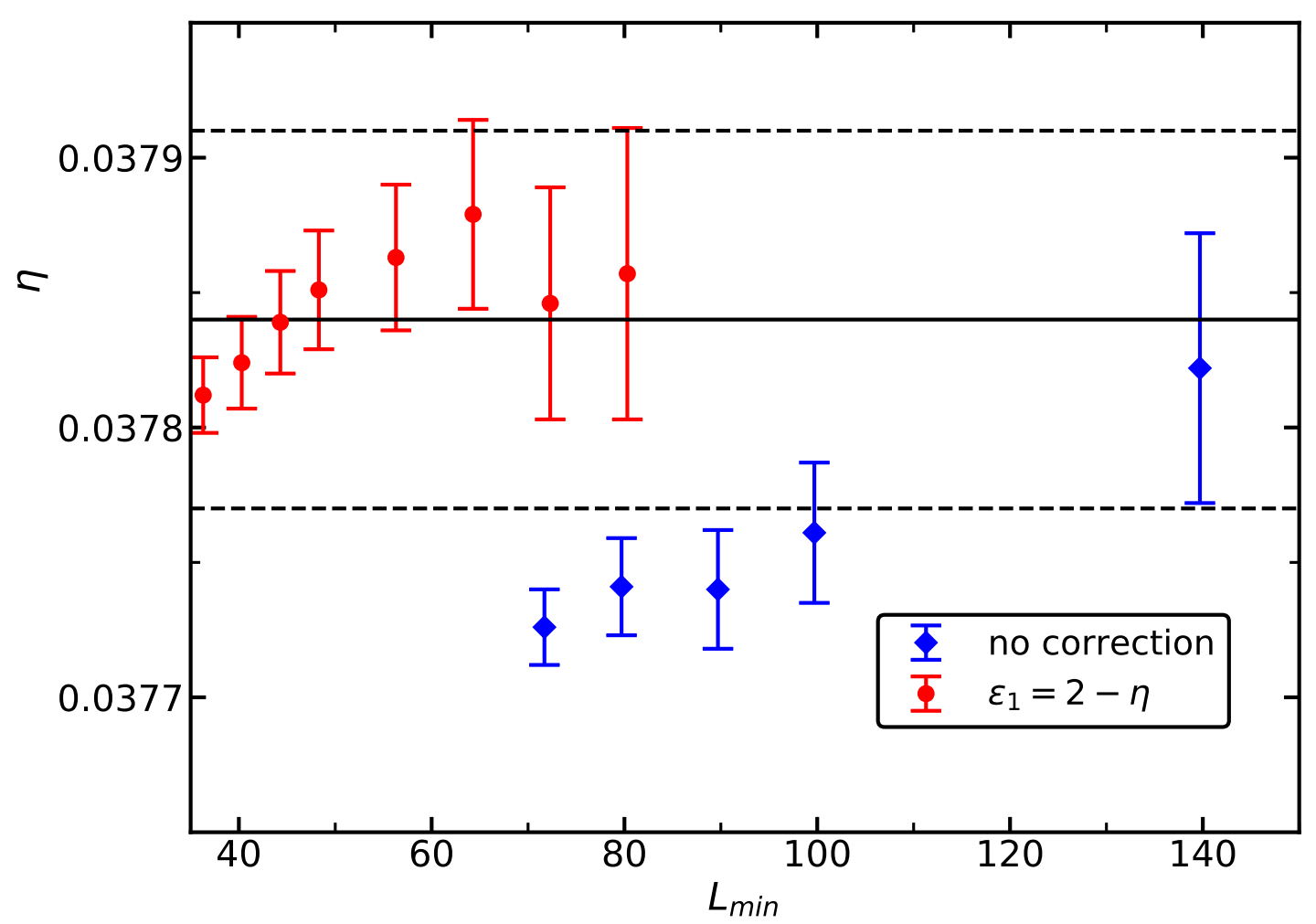

FIG. 10. We plot the estimates of $\eta$ obtained from fitting the improved magnetic susceptibility at $\xi_{2 n d} / L=0.56404$ as a function of the minimal lattice size $L_{\min }$ that is taken into account. Data for $D=2.05$ and $D=2.1$ are used. To make the figure more readable, we have shifted the values of $L_{\min }$ slightly. Only results for $Q>0.01$ are given. Either no correction or a term corresponding to the analytic background is used in the ansatz. The solid line gives our preliminary estimate of $\eta$ and the dashed lines indicate the error bar.

\section{SUMMARY AND DISCUSSION}

We have studied the generalized icosahedral model on the simple cubic lattice. In this model, the field variable might take a normalized vertex of the icosahedron as value. Analogous to the Blume-Capel model, in addition $(0,0,0)$ is allowed. The density of the $(0,0,0)$ sites is governed by the parameter $D$ of the reduced Hamiltonian. For a certain range of $D$, the model undergoes a second-order phase transition. At the critical line, the symmetry is enhanced to $O(3)$. Hence the transition belongs to the universality class of the three-dimensional Heisenberg model. In the Appendix $\mathrm{B}$ we find that a perturbation of the $O(3)$-invariant fixed point with the symmetry of the icosahedron is related with the irrelevant 
RG-eigenvalue $y_{i c o}=-2.19(2)$. On the critical line, the amplitude of leading corrections to scaling depends of the parameter $D$. Numerically we find that for $D^{*}=2.08(2)$ this amplitude vanishes. Based on a finite size scaling analysis of phenomenological couplings, such as the Binder cumulant, their slopes and the magnetic susceptibility we arrive at accurate estimates of the critical exponents $\nu$ and $\eta$ and the correction exponent $\omega$. In Appendix A we analyze data obtained for the three-component $\phi^{4}$ model on the simple cubic lattice, leading to consistent results for the exponents $\nu$ and $\eta$, confirming that both models share the same universality class. The precision of our results clearly surpasses that of experiments. However one should note that there had been theoretical advances in recent years made by different methods. Here our results serve as benchmark. In the introduction, in table I] we confront our results with ones given in the literature. Comparing with the $\epsilon$-expansion, we find significant deviations. The estimates obtained by using the conformal bootstrap method [17] and the recent implementation of the functional renormalization group method [18] are consistent with but less precise than ours.

Our precise estimates of the inverse critical temperature for various values of $D$ and $\lambda$ for the generalized icosahedral model and the $\phi^{4}$ model, respectively, might serve as input for studies focussing on other properties of these models. In particular we intend to compute the structure constants using a similar approach as in ref. [36] for the Ising universality class. Furthermore it would be interesting to investigate the symmetry properties of the icosahedral model in the low-temperature phase.

Our motivation to study the icosahedral model is of technical nature. In order to save the field variable at one site only 4 bits are needed. For practical reasons, in our program a 8 bit char variable is used. Furthermore, probabilities needed for the Metropolis and the cluster update can be computed and tabulated at the beginning of the simulation. For our implementation we find a speed up by roughly a factor of three compared with the $\phi^{4}$ model studied for example in refs. [8 10]. This advantage is partially abrogated by the correction $\propto L^{y_{i c o}}$ that is not present in a model with $O(3)$ symmetry at the microscopic level. Note that the situation is different for the $(q+1)$-state clock model studied in ref. [7]. In this case the irrelevant exponent $y_{q}$ is rapidly decreasing with $q$. In ref. [7] we focused on $q=8$, where $y_{q=8}=-5.278(9)$, see ref. [23]. Hence the correction can be ignored in the analysis of the data, meaning that we have the technical advantage without a downside. 


\section{ACKNOWLEDGEMENT}

This work was supported by the Deutsche Forschungsgemeinschaft (DFG) under grant No HA 3150/5-1.

\section{Appendix A: The $\phi^{4}$ model on the lattice}

The $\phi^{4}$ model on the simple cubic lattice is defined by the reduced Hamiltonian

$$
\mathcal{H}_{\phi^{4}}=-\beta \sum_{<x y>} \vec{\phi}_{x} \cdot \vec{\phi}_{y}+\sum_{x}\left[\vec{\phi}_{x}^{2}+\lambda\left(\vec{\phi}_{x}^{2}-1\right)^{2}\right],
$$

where $\vec{\phi}_{x} \in \mathbb{R}^{N}$ with $N=3$ in our case. We performed simulations for $\lambda=5$ and 5.2. Note that $\lambda^{*}=5.2(4)$, eq. (B13) of ref. [10]. We simulated at $\beta=0.6875638$ and 0.687985 in the case of $\lambda=5$ and 5.2, respectively. These are the estimates of $\beta_{c}$ obtained in ref. [9] and in preliminary simulations, respectively. The simulations are organized in a similar fashion as for the generalized icosahedral model. For $\lambda=5.2$ we have simulated the linear lattice sizes $L=8,9, \ldots, 20,22, \ldots, 30,34,40,50,60,80,100,140,200$, and 300 . The number of measurements decreases with increasing lattice size. Up to $L=19$ we performed about $3 \times 10^{9}$ measurements. For $L=300$ we performed $3.75 \times 10^{7}$ measurements. In total we spent about 13.5 years of $\mathrm{CPU}$ time on these simulations. In the case of $\lambda=5.0$ we performed simulations for fewer lattice sizes. We simulated at $L=8,10, \ldots, 30,34,40,50$, 60, 80, 100 and 140. In total we spent about 5.5 years of CPU time on these simulations.

\section{a. The inverse critical temperature}

First we determine the inverse critical temperature by analyzing the improved phenomenological coupling $Z_{a} / Z_{p}+0.575 U_{4}$. We fit our data with the ansatz

$$
R\left(L, \beta_{c}\right)=R^{*}+c L^{-2}
$$

where we use the estimate $\left(Z_{a} / Z_{p}+0.575 U_{4}\right)^{*}=0.84987(3)$ that we obtained from the analysis of the data for the icosahedral model in section IVA. As final result we get

$$
\begin{aligned}
& \beta_{c}(\lambda=5.0)=0.68756127(13)[6], \\
& \beta_{c}(\lambda=5.2)=0.68798521(8)[3],
\end{aligned}
$$

where the error in [] is due to the uncertainty of $\left(Z_{a} / Z_{p}+0.575 U_{4}\right)^{*}$. 


\section{b. $\quad$ The improved model}

Here we study the behavior of $U_{4}$ at $\xi_{2 n d} / L=0.56404$ or $Z_{a} / Z_{p}=0.19477$.

We perform fits similar to those performed in section IVC. Here we only include the data obtained for $\lambda=5.0$ and 5.2. Furthermore we fix the value of $\bar{U}_{4}^{*}$ to that obtained in section IVC. For $\xi_{2 n d} / L=0.56404$ we get $\lambda^{*}=5.19(2)[6]$, while for $Z_{a} / Z_{p}=0.19477$ we get $\lambda^{*}=5.14(2)[6]$, where the error in [] is due to the uncertainty of $\bar{U}_{4}^{*}$. Our final estimate

$$
\lambda^{*}=5.17(11)
$$

is chosen such that both estimates, including their errors are covered.

\section{c. Finite size scaling estimate of $\nu$}

Here we performed an analysis similar to that for the icosahedral model in section IVD. The data for $D=2.05$ and 2.1 for the icosahedral model are replaced by those for $\lambda=5.0$ and 5.2. Below we discuss results obtained by analyzing improved slopes at $Z_{a} / Z_{p}=0.19477$. The corresponding results for $\xi_{2 n d} / L=0.56404$ differ only by little.

In Fig. 11 we give estimates of $y_{t}$ obtained by using an ansatz without correction term, eq. (30). Similar to Fig. 5 we find that for small $L_{\min }$ the estimates obtained from different phenomenological couplings do not agree within their respective error bars. As our final estimate we take

$$
y_{t}=1.4052(5)
$$

corresponding to $\nu=0.71164(25)$. This estimate is consistent with results obtained for some range of $L_{\min }$ for each of the three phenomenological couplings.

\section{d. Finite size scaling estimate of the exponent $\eta$}

We performed joint fits of the data for the magnetic susceptibility at $\lambda=5.0$ and 5.2 by using the ansatz (35) or the ansatz (36) using either $\epsilon_{2}=2.02$ or 4 . We analyzed both the improved magnetic susceptibility at $Z_{a} / Z_{p}=0.19477$ and $\xi_{2 n d} / L=0.56404$. The

results of such fits for $Z_{a} / Z_{p}=0.19477$ are plotted in Fig. 12. Our preliminary estimate $\eta=0.03784(8)$ is chosen such that the estimates of $\eta$ obtained by using the three different 


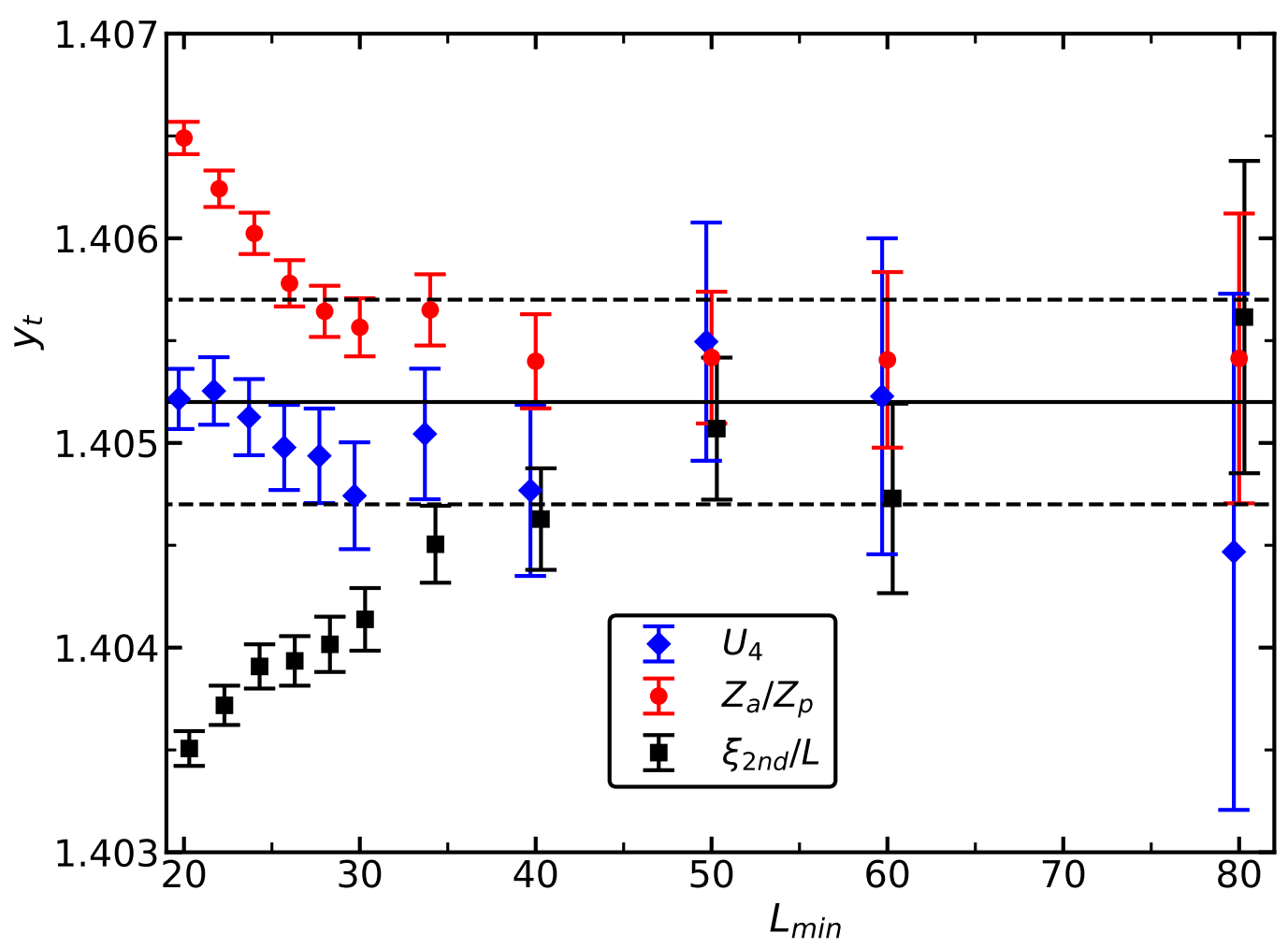

FIG. 11. We plot the estimates of $y_{t}$ obtained by fitting the improved slopes of phenomenological couplings at $Z_{a} / Z_{p}=0.19477$ by using the ansatz (30) without corrections as a function of $L_{\min }$. Data for the $\phi^{4}$ model at $\lambda=5$ and $\lambda=5.2$ are analysed jointly. Only results for $Q>0.01$ are given. To make the figure more readable, we have shifted the values of $L_{m i n}$ slightly. The solid line indicates the final estimate that we obtain from this set of fits. The dashed lines give the error bar.

ansätze are contained in the range $0.03784 \pm 0.00008$ for some range of the minimal lattice size $L_{\min }$. Performing a similar analysis for $\xi_{2 n d} / L=0.56404$ we arrive at the slightly smaller estimate $\eta=0.03780(8)$. As the final estimate we quote

$$
\eta=0.03782(10)
$$

which covers both the estimates obtained from the data for fixing $Z_{a} / Z_{p}=0.19477$ and $\xi_{2 n d} / L=0.56404$. 


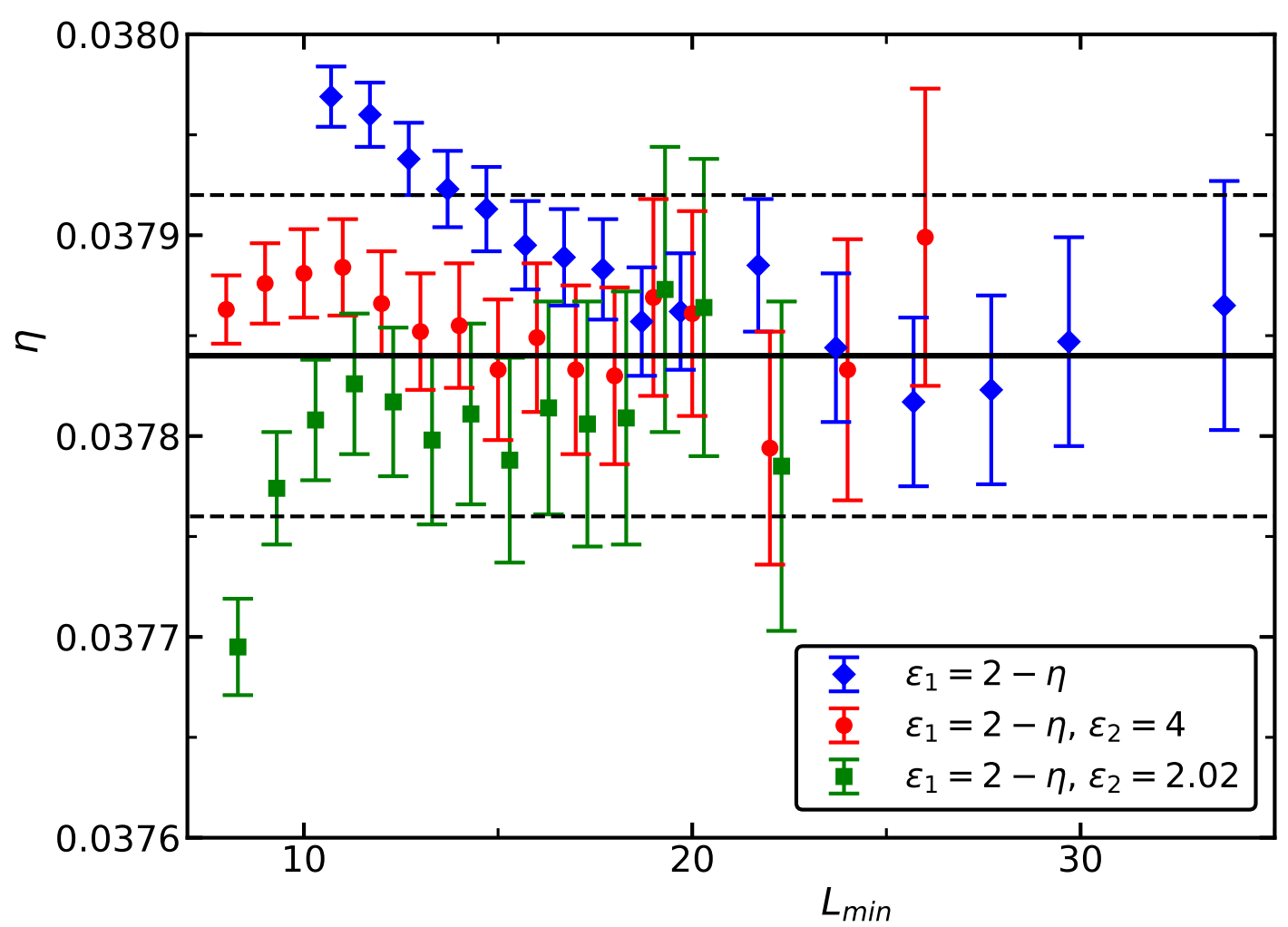

FIG. 12. We plot the estimates of $\eta$ obtained by fitting the improved magnetic susceptibility $\chi$ at $Z_{a} / Z_{p}=0.19477$ for $\lambda=5.0$ and 5.2 jointly. The effective correction exponents given in the legend refer to the ansätze (35/36). In the fits all lattice sizes $L \geq L_{\min }$ are taken into account. To make the figure more readable, we have shifted the values of $L_{\min }$ slightly. The solid line gives the preliminary estimate that we obtain from this set of fits. The dashed lines give the error bar.

\section{e. Reanalysis of the high temperature series expansion}

Our more precise estimates of $\lambda^{*}$ and the more accurate estimate of $\beta_{c}$ at $\lambda=5.0$ are used to bias the analysis of the high temperature series performed in ref. [9]. We start from tables XXV and XXVI in appendix B of ref. [9]. For $\lambda=4,4.5$ and 5 the high temperature series of the magnetic susceptibility and the second moment correlation length is analyzed by using biased integral approximants [19]. To this end, the estimates of $\beta_{c}$ given in eqs. (B5,B6,B7) of [9] are used. Let us discuss the details of our reanalysis at the example of the exponent $\nu$, given in table XXVI of appendix B. The estimates of $\nu$ carry two types of error estimates: the number given () is obtained from the spread of different approximants, while the number given in [] is due to the uncertainty of the estimate of $\beta_{c}$. 
It was obtained by reanalyzing the series for $\beta_{c} \pm \Delta \beta_{c}$, where $\Delta \beta_{c}$ is the estimate of the error of $\beta_{c}$. Note that the value obtained for the exponent is increasing with an increasing estimate of $\beta_{c}$. Hence the new estimate of the exponent is given by

$$
\nu_{\text {new }}=\nu_{\text {old }}+\left(\beta_{c, \text { new }}-\beta_{c, \text { old }}\right) \frac{\Delta \nu}{\Delta \beta_{c}} .
$$

Here "new" refers to the present work, while" old" refers to ref. [9]. $\Delta \beta_{c}$ is the error estimate of $\beta_{c}$ in ref. [9] and $\Delta \nu$ refers to the number given in [] in table XXVI of ref. [9].

Shifting the estimates of $\nu$ for $\lambda=5$ for the approximants bIA1 and bIA2 we arrive at $\nu=0.71141(5)[1]$ and $0.71144(6)[1]$, respectively. For $\lambda=4.5$, using the estimate of $\beta_{c}$ obtained in ref. [10], we arrive at $\nu=0.71103(3)[4]$ and 0.71102(6)[4]. Finally, extrapolating to $\lambda^{*}=5.17(11)$ we arrive at

$$
\nu=0.7116(2)
$$

Performing a similar analysis for the exponent of the magnetic susceptibility we arrive at $\gamma=1.3965(3)$. Note that in ref. [9] $\gamma=1.3960(9)$ is quoted. Plugging in $\lambda^{*}=5.17(11)$ into eq. (19) of ref. [9]

$$
\eta \nu=0.02665(18)+0.00035(\lambda-4.5)
$$

we arrive at

$$
\eta=0.0378(3)
$$

where we took into account the uncertainty of $\lambda^{*}$ and $\nu$.

\section{Appendix B: The correction exponent $\omega_{i c o}$}

We consider the quantity

$$
q=\frac{\left\langle\max _{j} \vec{v}_{j} \cdot \vec{m}\right\rangle}{\langle|\vec{m}|\rangle}
$$

where $\vec{m}=\sum_{x} \vec{s}_{x}$ is the magnetization of a given configuration, and $\vec{v}_{j}$, with $j=1,2, \ldots, 12$ are the twelve possible values of the spin with unit length. Alternatively, one might define a quantity based of the polynomial given in ref. [20]. For an $O(3)$-invariant distribution of $\vec{m}$ the value of $q$ can be easily computed by using numerical integration. We get

$$
q^{*}=0.915874306174
$$


The deviation from an $O(3)$-invariant distribution is now quantified by

$$
\bar{q}=q-q^{*}
$$

We computed $q$ at either $Z_{a} / Z_{p}=0.19477$ or $\xi_{2 n d} / L=0.56404$. It turns out that the numbers for $Z_{a} / Z_{p}=0.19477$ and $\xi_{2 n d} / L=0.56404$ are very similar and the estimates of $\omega_{i c o}$ are essentially the same for these two cases. Therefore we restrict the discussion below on $Z_{a} / Z_{p}=0.19477$. We fitted $\bar{q}$ by using the ansätze

$$
\bar{q}=a L^{-\omega_{i c o}}
$$

and

$$
\bar{q}=a L^{-\omega_{i c o}}\left(1+c L^{-2}\right) .
$$

First we checked the effect of leading corrections to scaling. To this end we fitted our data for $D=\infty, 1.4$, and 1.0 using the ansatz (B4) and $L_{\text {min }}=12$ in all three cases. We get $\omega_{i c o}=2.237(13), 2.125(7)$, and 2.069(5) and $\chi^{2} /$ d.o.f. $=0.91,0.78$, and 2.22 for $D=\infty$, 1.4, and 1.0, respectively. We see a clear dependence of the result for $\omega_{i c o}$ on $D$. Note that for both $D=\infty$ and 1.4 we get an acceptable $\chi^{2} /$ d.o.f., while the estimates of $\omega_{i c o}$ are inconsistent.

Based on fits with the ansatz (20) discussed in section IVB we know that the modulus of the amplitude of leading corrections to scaling at $D=2.05$ and 2.1 is by about a factor of 30 smaller than for $D=\infty$ or 1.4. Therefore the effect on the estimate of $\omega_{i c o}$ should roughly be given by $[2.237(13)-2.125(7)] / 60$, which we might ignore in the following.

In Fig. 13 we plot the result of joint fits for $D=2.05$ and 2.1 of $\bar{q}$ at $Z_{a} / Z_{p}=0.19477$. The free parameters are $a(D=2.05), a(D=2.1)$, and $\omega_{i c o}$ for ansatz (B4). In the case of ansatz (B5) $c$ is an additional free parameter, where we assume $c$ to be the same for $D=2.05$ and 2.1 .

As our final estimate we take

$$
\omega_{i c o}=2.19(2)
$$

This estimate is chosen such that the estimates obtained by using the ansatz (B4) for $5 \leq L_{\text {min }} \leq 9$ and the ansatz $(\overline{\mathrm{B} 5})$ for $11 \leq L_{\text {min }} \leq 20$ including the respective error bars are covered. 


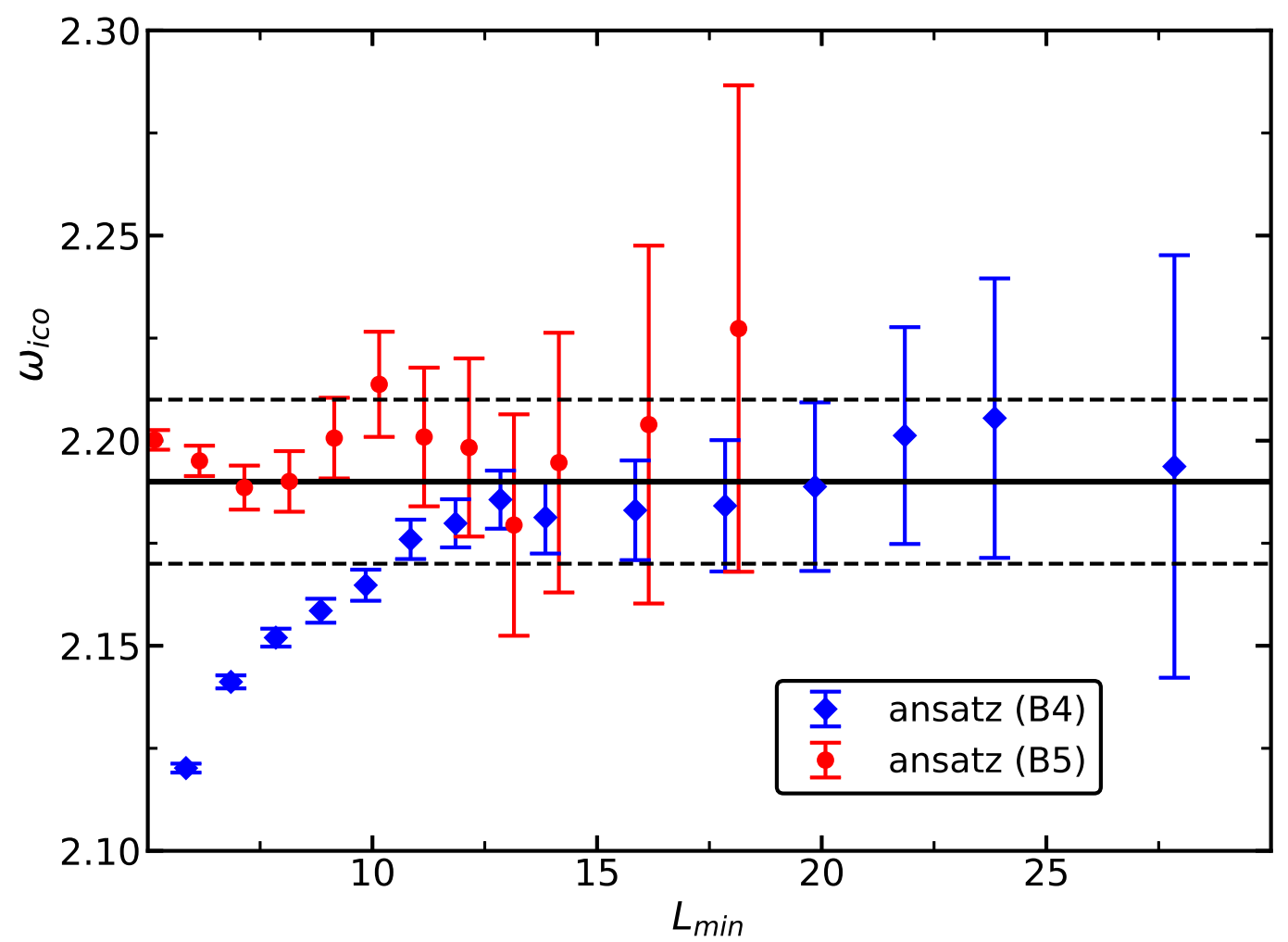

FIG. 13. We plot the estimate of $\omega_{i c o}$ obtained from fitting $\bar{q}$ at $Z_{a} / Z_{p}=0.19477$ as a function of the minimal lattice $L_{\text {min }}$ size that is taken into account. Data for $D=2.1$ and 2.05 are jointly analyzed by using the ansätze (B/B5) . For readability the values of $L_{\min }$ are slightly shifted. The solid line gives our final estimate $\omega_{i c o}=2.19$, while the dashed lines indicate the error.

[1] A. Pelissetto and E. Vicari, Critical Phenomena and Renormalization-Group Theory, arXiv:cond-mat/0012164], Phys. Rept. 368, 549 (2002).

[2] K. G. Wilson and J. Kogut, The renormalization group and the $\epsilon$-expansion, Phys. Rep. C 12, 75 (1974).

[3] M. E. Fisher, The renormalization group in the theory of critical behavior, Rev. Mod. Phys. 46, 597 (1974).

[4] M. E. Fisher, Renormalization group theory: Its basis and formulation in statistical physics, Rev. Mod. Phys. 70, 653 (1998).

[5] D. C. Rapaport, A discrete vector spin model, J. Phys. A: Math. Gen. bf 18, L667 (1985). 
[6] A. Margaritis, G. Odor and A Patkòs, Sequence of discrete spin models approximating the classical Heisenberg ferromagnet, J. Phys. A: Math. Gen. 20, 1917 (1987).

[7] M. Hasenbusch, Monte Carlo study of an improved clock model in three dimensions, arXiv:1910.05916], Phys. Rev. B 100, 224517 (2019).

[8] M. Hasenbusch, Eliminating leading corrections to scaling in the 3-dimensional $O(N)$ symmetric $\phi^{4}$ model: $N=3$ and 4, arXiv:cond-mat/0010463, J. Phys. A 34, 8221 (2001).

[9] M. Campostrini, P. Rossi, E. Vicari, M. Hasenbusch, and A. Pelissetto, Critical Exponents and Equation of State of the Three-Dimensional Heisenberg Universality Class, arXiv:cond-mat/0110336], Phys. Rev. B 65, 144520 (2002).

[10] M. Hasenbusch and E. Vicari, Anisotropic perturbations in three-dimensional $O(N)$ symmetric vector models, arXiv:1108.0491, Phys. Rev. B 84, 125136 (2011).

[11] A. Herrero, A. Oleaga, P. Manfrinetti, A. Provino, and A. Salazar, Critical behavior of the ferromagnetic transition in $\mathrm{GdSc}(\mathrm{Si}, \mathrm{Ge})$ intermetallic compounds, Intermetallics 101, 64 (2018).

[12] R. Guida and J. Zinn-Justin, Critical exponents of the $N$ vector model, arXiv:cond-mat/9803240], J. Phys. A 31, 8103 (1998).

[13] M. V. Kompaniets and E. Panzer, Minimally subtracted six-loop renormalization of $\phi^{4}-$ symmetric theory and critical exponents, arXiv:1705.06483, Phys. Rev. D 96, 036016 (2017).

[14] O. Schnetz, Numbers and Functions in Quantum Field Theory, arXiv:1606.08598, Phys. Rev. D 97, 085018 (2018).

[15] D. Simmons-Duffin, The Lightcone Bootstrap and the Spectrum of the 3d Ising CFT, [arXiv:1612.08471], JHEP 03 (2017) 086.

[16] S. M. Chester, W. Landry, J. Liu, D. Poland, D. Simmons-Duffin, N. Su, and A. Vichi, Carving out OPE space and precise $O(2)$ model critical exponents, arXiv:1912.03324], JHEP 06 (2020) 142 .

[17] F. Kos, D. Poland, D. Simmons-Duffin, and A. Vichi, Precision Islands in the Ising and $O(N)$ Models arXiv:1603.04436], JHEP 08 (2016) 036.

[18] G. De Polsi, I. Balog, M. Tissier, and N. Wschebor, Precision calculation of critical exponents in the $O(N)$ universality classes with the nonperturbative renormalization group, arXiv:2001.07525, Phys. Rev. E 101, 042113 (2020).

[19] For a review see, e.g., A. J. Guttmann, in Phase Transitions and Critical Phenomena, Vol. 13, edited by C. Domb and J. Lebowitz (Academic, New York, 1989). 
[20] S. Caracciolo, A. Montanari, and A. Pelissetto, Asymptotically free models and discrete nonAbelian groups, arXiv:hep-lat/0103017, Phys. Lett. B 513, 223 (2001).

[21] K. E. Newman and E. K. Riedel, Critical exponents by the scaling-field method: The isotropic $N$-vector model in three dimensions, Phys. Rev. B 30, 6615 (1984).

[22] M. Campostrini, A. Pelissetto, P. Rossi, and E. Vicari, Two-point correlation function of three-dimensional $O(N)$ models: The critical limit and anisotropy, arXiv:cond-mat/9705086, Phys. Rev. E 57, 184 (1998).

[23] D. Banerjee, S. Chandrasekharan, and D. Orlando, Conformal dimensions via large charge expansion, arXiv:1707.00711, Phys. Rev. Lett. 120, 061603 (2018).

[24] D. Banerjee, S. Chandrasekharan, D. Orlando, and S. Reffert, Conformal Dimensions in the Large Charge Sectors at the O(4) Wilson-Fisher Fixed Point, arXiv:1902.09542, Phys. Rev. Lett. 123, 051603 (2019).

[25] R. B. A. Zinati, A. Codello, and G. Gori, Platonic Field Theories, arXiv:1902.05328, JHEP04 (2019) 152.

[26] R.H. Swendsen and J.-S. Wang, Nonuniversal critical dynamics in Monte Carlo simulations, Phys. Rev. Lett. 58, 86 (1987).

[27] U. Wolff, Collective Monte Carlo Updating for Spin Systems, Phys. Rev. Lett. 62, 361 (1989).

[28] M. Hasenbusch, K. Pinn, S. Vinti, Critical Exponents of the 3D Ising Universality Class From Finite Size Scaling With Standard and Improved Actions, arXiv:hep-lat/9806012], Phys. Rev. B 59, 11471 (1999).

[29] M. Campostrini, M. Hasenbusch, A. Pelissetto, P. Rossi, and E. Vicari, Critical behavior of the three-dimensional XY universality class, arXiv:cond-mat/0010360, Phys. Rev. B 63, 214503 (2001).

[30] M. Saito and M. Matsumoto, "SIMD-oriented Fast Mersenne Twister: a 128-bit Pseudorandom Number Generator", in Monte Carlo and Quasi-Monte Carlo Methods 2006, edited by A. Keller, S. Heinrich, H. Niederreiter, (Springer, 2008); M. Saito, Masters thesis, Math. Dept., Graduate School of science, Hiroshima University, 2007. The source code of the program is provided at http://www.math.sci.hiroshima-u.ac.jp/ m-mat/MT/SFMT/index.html

[31] T. E. Oliphant, Python for Scientific Computing, Computing in Science \& Engineering 9, 10 (2007); E. Jones,E. Oliphant, P. Peterson, et al.,SciPy: Open Source Scientific Tools for Python, 2001-, http://www.scipy.org/, P. Virtanen, R. Gommers, T. E. Oliphant et al., 
SciPy 1.0: fundamental algorithms for scientific computing in Python, arXiv:1907.10121, Nature Methods 17, 261 (2020).

[32] J. J. Moré, B. S. Garbow, and K. E. Hillstrom, User Guide for MINPACK-1, Argonne National Laboratory Report ANL-80-74, Argonne, Ill., (1980); J. J. Moré, D. C. Sorensen, K. E. Hillstrom, and B. S. Garbow, The MINPACK Project, in Sources and Development of Mathematical Software, W. J. Cowell, ed., Prentice-Hall, 88 (1984).

[33] J. D. Hunter, "Matplotlib: A 2D Graphics Environment, Computing in Science \& Engineering 9, $90(2007)$.

[34] Y. Deng, H. W. J. Blöte, and M. P. Nightingale, Surface and bulk transitions in threedimensional $O(n)$ models, arXiv:cond-mat/0504173, Phys. Rev. E 72, 016128 (2005).

[35] M. Hasenbusch, F. Parisen Toldin, A. Pelissetto, and E. Vicari, Universality class of 3D sitediluted and bond-diluted Ising systems, arXiv:cond-mat/0611707, J. Stat. Mech.: Theory Exp. 2007, P02016.

[36] M. Hasenbusch, Two- and three-point functions at criticality: Monte Carlo simulations of the improved three-dimensional Blume-Capel model, arXiv:1711.10946], Phys. Rev. E 97 (2018) 012119 . 